\title{
Multi-morph eco-evolutionary dynamics in structured populations
}

\author{
Sébastien Lion ${ }^{1, *}$, Mike Boots ${ }^{2,3}$ and Akira Sasaki ${ }^{4,5}$
}

July 8, 2021

1. CEFE, CNRS, Univ Montpellier, EPHE, IRD, Univ Paul Valéry Montpellier 3. 1919, route de Mende, Montpellier, France.

Email: sebastien.lion@cefe.cnrs.fr. ORCID: 0000-0002-4081-0038.

2. Integrative Biology, University of California, Berkeley, USA. CA 94720.

Email: mboots@berkeley.edu. ORCID: 0000-0003-3763-6136.

3. Biosciences, University of Exeter, Penryn Campus, UK. TR10 9FE.

4. Department of Evolutionary Studies of Biosystems, The Graduate University of Advanced Studies, SOKENDAI, Hayama, Kanagawa 2400139, Japan.

Email: sasaki_akira@soken.ac.jp. ORCID: 0000-0003-3582-5865.

5 Evolution and Ecology Program, International Institute for Applied Systems Analysis, Schlosplatz 1, A-2361, Laxenburg, Austria.

* corresponding author

Keywords: quantitative genetics, adaptive dynamics, reproductive value, environmental feedbacks, time scales

\begin{abstract}
Our understanding of the evolution of quantitative traits in nature is still limited by the challenge of including realistic trait distributions in the context of frequency-dependent selection and ecological feedbacks. We develop a theoretical framework to analyse the dynamics of populations composed of several morphs and structured into distinct classes (e.g. age, size, habitats, infection status, species...). Our approach extends to class-structured populations a recently introduced "oligomorphic approximation" which bridges the gap between adaptive dynamics and quantitative genetics approaches and allows for the joint description of the dynamics of ecological variables and of the moments of multimodal trait distributions. We also introduce a new approximation to simplify the eco-evolutionary dynamics using reproductive values. This effectively extends Lande's univariate theorem not only to frequency- and density-dependent selection but also to multimodal trait distributions. We illustrate the effectiveness of this approach by applying it to the important conceptual case of two-habitat migration-selection models. In particular, we use our approach to predict the equilibrium trait distributions in a local adaptation model with asymmetric migration and habitat-specific mutational variance. We discuss the theoretical and practical implications of our results and sketch perspectives for future work.
\end{abstract}

Many experimental and empirical studies in evolutionary ecology aim at understanding how ecological processes affect how trait distributions change over time. This has motivated the development of quantitative genetics methods to analyse the dynamics of quantitative traits (Lande, 1979; Bulmer, 1992; Falconer, 1996; Walsh \& Lynch, 2018). Following Lande (1976, 1979, 1982)'s seminal work, most quantitative genetics models assume unimodal trait distributions and frequency-independent selection, leaving aside the problem of how multimodal distributions can be generated by frequency-dependent disruptive selection. Under these assumptions, dynamical equations for the mean and higher moments of a trait distribution can be derived for tightly clustered trait distributions (Barton \& Turelli, 1987; Turelli \& Barton, 1990; Barton \& Turelli, 1991). Assuming that trait distributions are narrowly localised around a single mean also allows one to incorporate frequency-dependence to some extent (Iwasa et al., 1991; Abrams et al., 1993), but many models rely on the more classical assumption that trait distributions are and remain normally distributed. However, empirical evidence of skewed (Bonamour et al., 2017) or multimodal distributions highlight the need for an alternative approach. 
A major limitation of current quantitative genetics theory is the reliance on simplified ecological scenarios that are not representative of the complexity of eco-evolutionary feedbacks in nature. This led to the development of adaptive dynamics theory, which, under the assumption that evolution is limited by rare mutations, provides a mathematical framework to study the interplay between ecological and evolutionary processes (Metz et al., 1992; Dieckmann \& Law, 1996; Metz et al., 1996; Geritz et al., 1998). Many authors have noted the similarities and subtle differences between AD and QG approaches under the assumptions of small mutational steps and narrow trait distributions respectively (Abrams et al., 1993; Abrams, 2001; Day, 2005; Lion, 2018c). However, there is a clear conceptual gap in the canonical approaches to AD and QG: while adaptive dynamics has been successful in taking into account environmental feedbacks and the emergence of polymorphism under frequency-dependent disruptive selection, it does so by assuming strong constraints on the mutation process and standing variation in the population. Recently, Sasaki \& Dieckmann (2011) proposed an alternative oligomorphic approach to bridge the gap between adaptive dynamics and quantitative genetics theory. The crux of the oligomorphic approximation is to decompose the trait distribution into a sum of narrow unimodal morph distributions and to derive the dynamics of the frequency, mean trait value and variance of each morph. Suitable moment closure approximations at the morph level yield a closed dynamical system. As such, the oligomorphic approximation can be seen as an extension of quantitative genetics theory to take into account eco-evolutionary feedbacks and polymorphic trait distributions.

The theoretical developments of Sasaki \& Dieckmann (2011) rely on a number of additional assumptions, notably single-locus haploid genetics, large populations sizes, and unstructured populations. In this paper, we retain the first two assumptions but investigate how class structure affects the dynamics of quantitative trait distributions under the oligomorphic approximation. As class structure is ubiquituous in biological populations, this is an important extension to Sasaki \& Dieckmann (2011)'s theory, allowing us to take into account the fact that individuals can be in distinct demographic, physiological or ecological states, such as different age groups, developmental stages, infection status, or habitats.

The paper is organised as follows. We first give a general decomposition of the trait distribution into different morphs in a population structured into distinct classes. We then show how, by assuming that each morph distribution is clustered around the morph mean, we can derive equations for the dynamics of class-specific morph frequencies, morph means, and morph variances using Taylor approximations of the vital rates describing between-classes transitions. We also apply recent theory on reproductive value (Lion, 2018a,b; Lion \& Gandon, 2021) to simplify the morph dynamics at the population level. In particular, we show that the dynamics of morph means take the form of a frequency-dependent extension of Lande's theorem (Lande, 1976, 1979, 1982; Barfield et al., 2011), where the marginal fitness effect on the vital rate from class $j$ to class $k$ is weighted by the morph's reproductive value in class $k$ (a measure of class quality) and by the fraction of morph- $i$ individuals that are in class $j$ (a measure of class quantity). Finally, we derive some simpler results for the important limit case of two-class models, and apply this general framework to two specific models describing the interplay between migration and selection in a population distributed over two habitats of distinct qualities coupled by migration. The first example is a two-habitat extension of the resource competition model analysed by Sasaki \& Dieckmann (2011). The second example revisits the local adaptation models analysed by Meszéna et al. (1997), Ronce \& Kirkpatrick (2001), Débarre et al. (2013), and Mirrahimi \& Gandon (2020), but our oligomorphic approach allows us to express their results in terms of the reproductive values of each habitat and to extend the analysis to asymmetric migration.

\section{Densities and trait distributions}

We consider a population of individuals characterised by a continuous phenotypic trait. The total density of individuals with trait value $z$ at time $t$ is $n(z, t)$ and, for simplicity, we denote the total density of individuals as $n(t)=\int n(z, t) \mathrm{d} z$ (with a slight abuse of notation). We further assume that the population is structured into $K$ discrete classes, which can for instance represent different age groups, developmental stages, or habitats. The density of individuals with trait value $z$ in class $k$ at time $t$ is $n^{k}(z, t)$. Similarly, we write $n^{k}(t)=\int n^{k}(z, t) \mathrm{d} z$ for the total density of individuals in class 
$k$ at $t$. See Table 1 for a description of the main notations in the paper.

\section{$1.1 \quad$ Full distributions}

The within- and across-class densities represent the raw statistics of the model. They can be used to define some useful distributions to analyse the eco-evolutionary dynamics of the population. At the ecological level, the class distribution can be defined as

$$
f^{k}(t)=\frac{n^{k}(t)}{n(t)}
$$

which represents the fraction of individuals that are in class $k$ at time $t$. Note that $\sum_{k} f^{k}(t)=1$, where the summation is over all classes, i.e. $1 \leq k \leq K$ (for simplicity, all summation limits will be implicit in this article).

At an evolutionary level, two trait distributions can be defined. The within-class trait distribution is

$$
\phi^{k}(z, t)=\frac{n^{k}(z, t)}{n^{k}(t)},
$$

which is the frequency of individuals with trait $z$ in class $k$ at time $t$. Averaging over classes yields the across-class trait distribution

$$
\phi(z, t)=\frac{n(z, t)}{n(t)} .
$$

It is easy to check that, as expected, $\int \phi^{k}(z, t) \mathrm{d} z=\int \phi(z, t) \mathrm{d} z=1$. Note that the class and trait distributions are linked through the relationship $\phi(z, t)=\sum_{k} \phi^{k}(z, t) f^{k}(t)$.

\subsection{Oligomorphic decomposition}

Up to now, we have made no assumption on the trait distribution in the population. With the notations defined so far, it is straightforward to produce a continuous-trait version of the Price equations derived in Lion (2018a), but our aim here is slightly different, because we want to make specific predictions on the dynamics of multimodal distributions. Following Sasaki \& Dieckmann (2011), we therefore assume that the trait distribution can be decomposed into $M$ morphs. At the within-class level, we write

$$
\phi^{k}(z, t)=\sum_{i} \phi_{i}^{k}(z, t) f_{i}^{k}(t)
$$

where $\phi_{i}^{k}(z, t)$ is the distribution of morph $i$ in class $k$ at time $t$, and $f_{i}^{k}(t)$ is the frequency of morph $i$ in class $k$. Note that $\int \phi_{i}^{k}(z, t) \mathrm{d} z=1$ and $\sum_{i} f_{i}^{k}(t)=1$, where the summation is implicitly over all morphs (i.e. $1 \leq i \leq M$ ).

Equation (4) is a class-specific version of equation (5) in Sasaki \& Dieckmann (2011). Biologically it means that the full distribution $\phi^{k}(z, t)$ can be decomposed into a sum of morph distributions, $\phi_{i}^{k}(z, t)$, each weighted by the morph frequency, $f_{i}^{k}(t)$. Under the assumption that the morph distributions are sufficiently narrow, the dynamics of the full distribution can then be approximated by the dynamics of morph-specific statistics, such as the morph means and variances, and morph frequencies. Figure 1 gives a graphical illustration of the oligomorphic decomposition using a two-class, two-morph example.

\subsection{Morph moments}

From the distributions $\phi^{k}(z, t)$, we can calculate class-specific moments, such as $\bar{z}^{k}(t)$, the mean trait value in class $k$ at time $t$, and $V^{k}(t)$, the trait variance in class $k$ at time $t$. Similarly, morph-specific moments can be calculated from the distributions $\phi_{i}^{k}(z, t)$. For instance, the mean trait value of morph $i$ in class $k$ at $t$ is $\bar{z}_{i}^{k}(t)$, and the trait variance of morph $i$ in class $k$ at $t$ is $V_{i}^{k}(t)$.

Under suitable moment closure approximations at the morph level, a morph can then be characterised by its relative abundance (e.g. its frequency in each class, $f_{i}^{k}$ ), its position (e.g. the class-specific morph mean $\bar{z}_{i}^{k}$ ), and its width (e.g. the class-specific morph standard deviation $\sqrt{V_{i}^{k}}$ ). Equation (4) 
Table 1: Definition of mathematical symbols used in the text

\begin{tabular}{lll}
\hline Symbol & Definition & Description \\
\hline \hline$n^{k}(z, t)$ & & Density of individuals with trait $z$ in class $k$ at $t$ \\
$n^{k}(t)$ & & Total density of individuals in class $k$ at $t$ \\
$n(z, t)$ & $=\sum_{k} n^{k}(z, t)$ & Density of individuals with trait $z$ at $t$ \\
$n(t)$ & $=\sum_{k} n^{k}(t)$ & Total density of individuals at $t$ \\
\hline$f^{k}(t)$ & $=n^{k}(t) / n(t)$ & Fraction of individuals in class $k$ at $t$ \\
$\phi^{k}(z, t)$ & $=n^{k}(z, t) / n^{k}(t)$ & Frequency of trait $z$ in class $k$ at $t$ \\
$\phi(z, t)$ & $=n(z, t) / n(t)$ & Total frequency of trait $z$ at time $t$ \\
$f_{i}^{k}(t)$ & & Morph frequencies within class $k$ at $t$ \\
$\phi_{i}^{k}(z, t)$ & & Morph distributions within class $k$ at $t$ \\
\hline $\bar{z}^{k}(t)$ & $=\int z \phi^{k}(z, t) \mathrm{d} z$ & Mean trait value in class $k$ at $t$ \\
$\bar{z}(t)$ & $=\int z \phi(z, t) \mathrm{d} z$ & Mean trait value at $t$ in the whole population. \\
$\bar{z}_{i}^{k}(t)$ & $=\int z \phi_{i}^{j}(z, t) \mathrm{d} z$ & Mean trait value of morph $i$ in class $k$ at $t$ \\
\hline$V^{k}(t)$ & $=\int\left[z-\bar{z}^{k}(t)\right]^{2} \phi^{k}(z, t) \mathrm{d} z$ & Trait variance in class $k$ at $t$ \\
$V(t)$ & $=\int[z-\bar{z}(t)]^{2} \phi(z, t) \mathrm{d} z$ & Trait variance at $t$ in the whole population \\
$V_{i}^{k}(t)$ & $=\int\left[z-\bar{z}_{i}^{k}(t)\right]^{2} \phi_{i}^{k}(z, t) \mathrm{d} z$ & Morph variance in class $k$ at $t$ \\
\hline$u_{i}^{k}(t)$ & & Freq. of class- $k$ individuals among morph- $i$ individuals \\
$v_{i}^{k}(t)$ & & Morph-specific individual reproductive values in class $k$ \\
$c_{i}^{k}(t)$ & $=v_{i}^{k} u_{i}^{k}$ & Morph-specific class reproductive values in class $k$ \\
\hline$\phi_{i}(z, t)$ & $=\sum_{k} \phi_{i}^{k}(z, t) u_{i}^{k}(t)$ & Morph distribution at the population level at $t$ (with \\
$\widetilde{\phi}_{i}(z, t)$ & $=\sum_{k} \phi_{i}^{k}(z, t) c_{i}^{k}(t)$ & moments $\left.\bar{z}_{i}, V_{i}, \ldots\right)$ \\
& & RV-weighted morph distribution at the population level \\
\hline $\mathbf{R}(z)$ & & at $t\left(\right.$ with moments $\left.\widetilde{z}_{i}, \widetilde{V}_{i}, \ldots\right)$ \\
$\overline{\mathbf{R}}^{k}$ & & Matrix of transition rates $r^{k j}(z)(1 \leq k, j \leq K)$ \\
$\overline{\mathbf{R}}_{i}$ & & Matrix of average transition rates $\bar{r}^{k j}$ \\
\hline
\end{tabular}

allows us to make connections between population-level moments and morph-specific moments. See Table 1 for explicit definitions of the population-level and morph-specific moments, as well as figure 1 for a graphical summary of the notations.

\subsection{Notational conventions}

To simplify the notations, a number of conventions will be used throughout the paper. First, classes will be identified by superscripts and morphs by subscripts. For classes, we use the superscripts $j$ or $k$, so that an implicit summation over $k$ means that $k$ takes values between 1 and $K$. For morphs, we use the subscript $i$, which thus takes values between 1 and $M$. The symbol $\ell$ will be used either for classes or morphs, when needed. Second, whenever it is clear from the context, we shall drop the dependency on time, writing e.g. $f^{k}$ instead of $f^{k}(t)$.

\section{Dynamics and separation of time scales}

Having defined the statistics we need to describe the state of the population at a given time, we now turn to their dynamics. Figure 1 illustrates how a multimodal trait distribution can be decomposed into a mixture of unimodal morph distributions. By tracking the dynamics of these morph distributions, we can understand how the various peaks of the multimodal distribution move and change over time. To derive these dynamics, we first specify the rates associated with the different events of the life cycle, then we calculate an approximation of these rates under the assumption that the morph distributions are sufficiently narrow. 


\section{(a) Ecological variables}
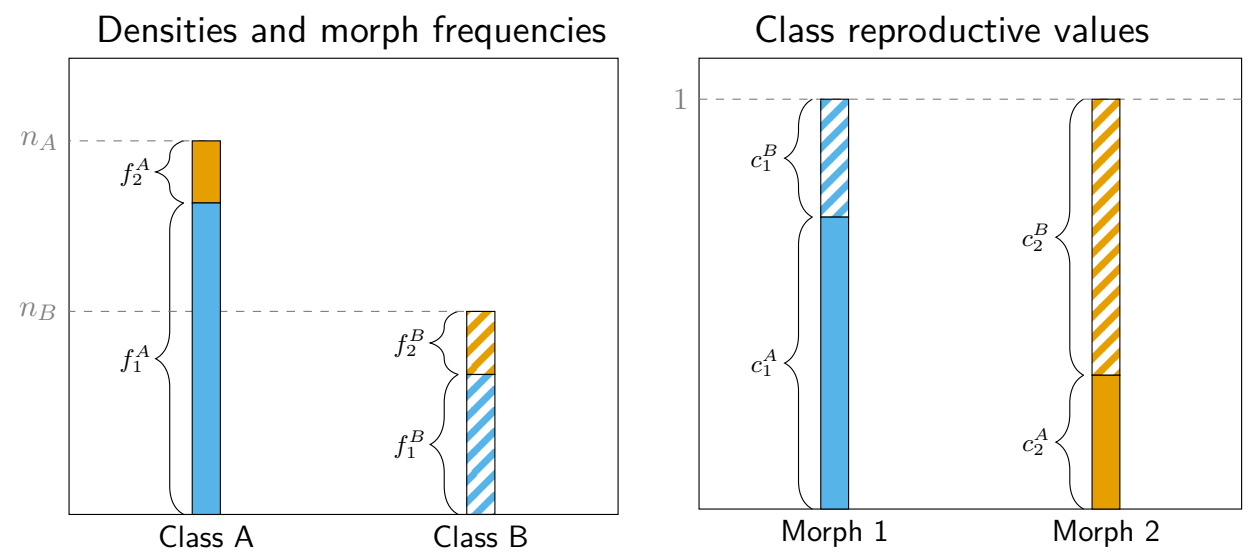

(b) Trait distributions

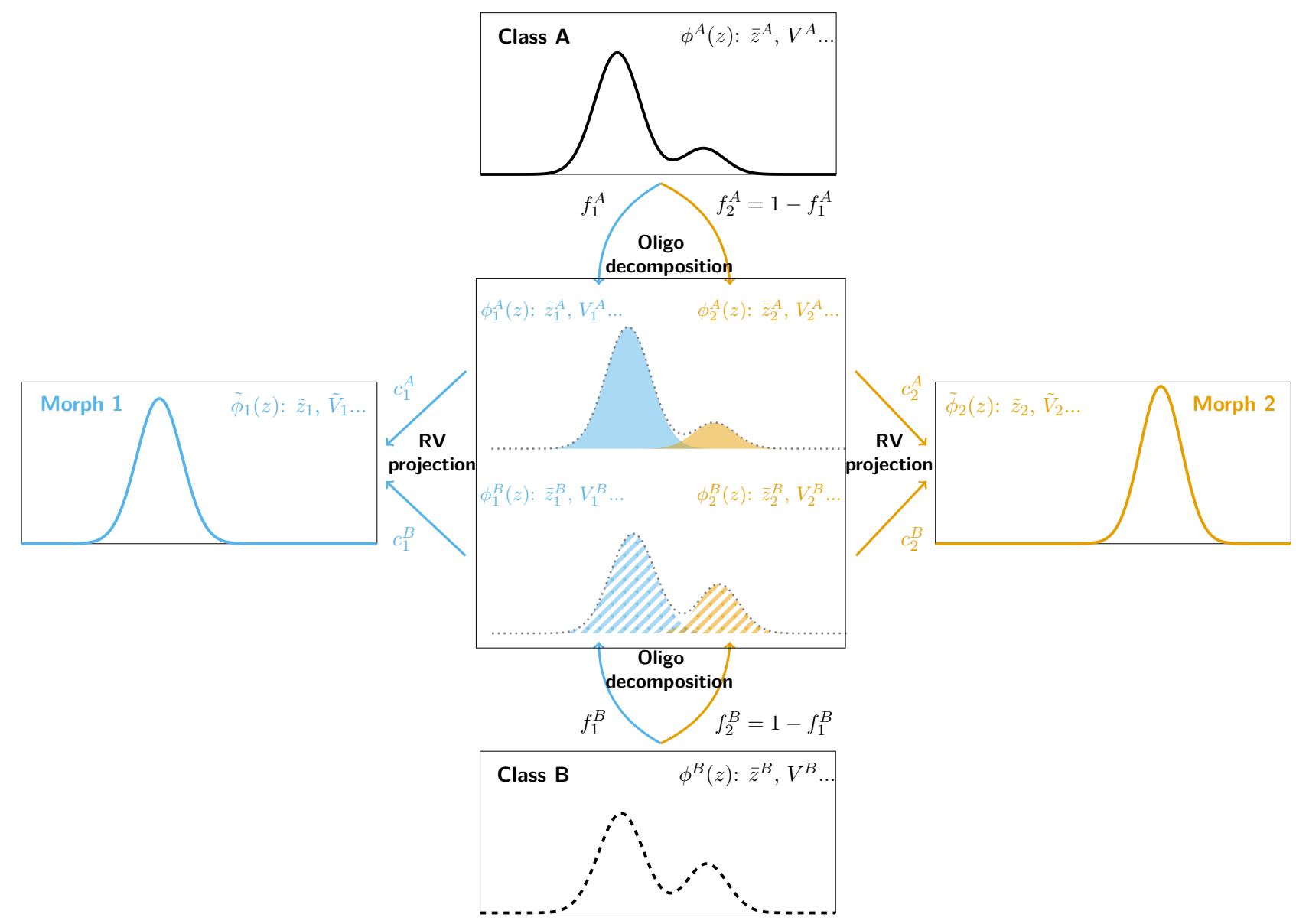

Figure 1: Summary of the notations and approach using a two-class, two-morph example. Panel (a) shows the fast variables, which change on the ecological time scale. These are the densities of individuals in each class, $n^{A}(t)$ and $n^{B}(t)$, the morph frequencies in each class, $f_{i}^{k}(t)$, and the morphspecific class reproductive values, $c_{i}^{k}(t)$. In the simulation snapshot used to plot these graphs, morph 1 is relatively more abundant within class $\mathrm{B}\left(f_{1}^{B}>f_{2}^{B}\right)$, but has a lower class reproductive value $\left(c_{1}^{B}<c_{2}^{B}\right)$. Panel (b) shows the trait distributions, which change on the slow, evolutionary time scales. The trait distribution in class $\mathrm{A}, \phi^{A}(z, t)$, can be decomposed into a mixture of class-specific morph distributions, $\phi_{i}^{A}(z, t)$, weighted by the class-specific morph frequencies $f_{i}^{A}(t)$. Note that, to better illustrate the decomposition, the shaded areas represent $f_{i}^{k}(t) \phi_{i}^{k}(z, t)$, and not the distributions $\phi_{i}^{k}(z, t)$. This oligomorphic decomposition can also be applied to class $B$. On the slow time scale, the relevant aggregate distributions at the morph level are the RV-weighted morph distributions $\widetilde{\phi}_{i}(z, t)$. Note that all graphs have the same axis limits. 


\section{$2.1 \quad$ Vital rates}

At a general level, the vital rates are defined by functions $r^{j k}(z, E(t))$, which give the rate of production of individuals in class $j$ by an individual in class $k$ with trait $z$ at time $t$. The variable $E(t)$ represents the environmental feedback, which collects all ecological variables needed to calculate the reproduction and survival of individuals (Metz et al., 1992; Mylius \& Diekmann, 1995; Metz et al., 2008; Lion, 2018c). For clonally reproducing organisms, this is sufficient to calculate the dynamics of the density $n^{j}(z, t)$, as follows

$$
\frac{\mathrm{d} n^{j}(z)}{\mathrm{d} t}=\sum_{k} r^{j k}(z, E(t)) n^{k}(z)
$$

In the remainder of the manuscript, all operations on the vital rates $r^{j k}(z, E(t))$ will be partial derivatives or integration with respect to the first argument. Hence, we shall drop the dependency on environmental feedback and write simply $r^{j k}(z)$ (and $\mathbf{R}(z)$ for the matrix of vital rates). However, it must be kept in mind that this notation does not imply density-independent or frequency-independent selection. Our formalism can be readily applied to scenarios where the vital rates depend on the density of conspecifics or other species, on the trait distribution, or on other biotic or abiotic ecological variables (see e.g. Sasaki \& Dieckmann (2011) and Lion (2018c)). Below, we will briefly illustrate this flexibility using a resource competition model.

\subsection{Oligomorphic approximation}

The crux of the oligomorphic approximation is to assume that the morph distribution is tightly clustered around its mean, that is the standard deviation of the distribution is proportional to a small parameter $\varepsilon$ (Sasaki \& Dieckmann, 2011). In a class-structured model, this means that the quantity $\xi_{i}^{k}=z-\bar{z}_{i}^{k}$ is small, and we write $\xi_{i}^{k}=O(\varepsilon)$. A simple Taylor expansion of the vital rates $r^{j k}(z)$ around the within-class morph mean $\bar{z}_{i}^{k}$ yields

$$
r^{j k}(z)=r^{j k}\left(\bar{z}_{i}^{k}\right)+\left.\xi_{i}^{k} \frac{\partial r^{j k}}{\partial z}\right|_{z=\bar{z}_{i}^{k}}+\left.\frac{1}{2}\left(\xi_{i}^{k}\right)^{2} \frac{\partial^{2} r^{j k}}{\partial z^{2}}\right|_{z=\bar{z}_{i}^{k}}+O\left(\varepsilon^{3}\right)
$$

Integrating over the distribution $\phi_{i}^{k}(z)$ yields an approximation for the average vital rates of morph $i$, in terms of the morph-specific mean and variances $\bar{z}_{i}^{k}$ and $V_{i}^{k}$. We have

$$
\bar{r}_{i}^{j k}=r^{j k}\left(\bar{z}_{i}^{k}\right)+\left.\frac{1}{2} V_{i}^{k} \frac{\partial^{2} r^{j k}}{\partial z^{2}}\right|_{z=\bar{z}_{i}^{k}}+O\left(\varepsilon^{4}\right)
$$

Similarly, averaging over the distribution $\phi^{k}(z)$ yields the average vital rates $\bar{r}^{j k}$, which can be decomposed in terms of morph averages as $\bar{r}^{j k}=\sum_{i} \bar{r}_{i}^{j k} f_{i}^{k}$.

\subsection{Separation of time scales}

The oligomorphic approximation gives rise to a separation of time scales between ecological and evolutionary variables. Specifically, the dynamics of the densities $n^{k}$, class frequencies $f^{k}$ and morph frequencies $f_{i}^{k}$ are all $O(1)$ so that these can be treated as fast variables. On the other hand, the dynamics of morph means and variances are at least $O(\varepsilon)$ and $O\left(\varepsilon^{2}\right)$, repectively, so the morph moments change on slower time scales. It is important to realise that this does not mean that there is no feedback between ecology and evolution, and in fact this approximation can be used to study situations where rapid evolution is fuelled by a large standing variance at the population level (for instance if we have two morphs with very different mean trait values), while assuming that the standing variation in each morph remains small.

This separation of time scales dictates the following step-by-step approach when analysing the dynamics of polymorphic populations. First, we derive the dynamics of the ecological variables and morph frequencies, for fixed values of the morph means and variances. The resulting equations are reminiscent of those governing allele frequency change in classical population genetics models. Second, 
we relax the assumption that morph means are fixed, and derive the dynamics of the morph means. Because the morph variances change on a slower time scale than the morph means, we can still assume at this stage that morph variances are fixed, as classically assumed in quantitative genetics. Finally, we relax this assumption, derive the dynamics of morph variances, and introduce a moment closure approximation at the morph level to close the system.

\section{Dynamics of ecological variables and morph frequencies}

In this section, we derive the dynamics of the fast variables, which are the class densities $n^{k}(t)$ and class-specific morph frequencies $f_{i}^{k}(t)$. We also introduce the idea of weighting each class by its reproductive value in order to calculate the net effect of selection on the change of frequency of a given morph.

\subsection{Dynamics of densities}

Collecting all the class densities $n^{k}(t)$ in a vector $\mathbf{n}$, we can write

$$
\frac{\mathrm{d} \mathbf{n}}{\mathrm{d} t}=\overline{\mathbf{R}} \mathbf{n}
$$

where $\overline{\mathbf{R}}$ is the matrix of average vital rates $\bar{r}^{j k}$. Using approximation (7), we have $\bar{r}^{j k}=\sum_{i} f_{i}^{k} r^{j k}\left(\bar{z}_{i}^{k}\right)+$ $O\left(\varepsilon^{2}\right)$, so the dynamics of class densities only depend, to zeroth order, on the morph frequencies and means.

Similarly, the vector of class frequencies $\mathbf{f}=\mathbf{n} / n$ has the following dynamics (Lion, 2018a)

$$
\frac{\mathrm{d} \mathbf{f}}{\mathrm{d} t}=\overline{\mathbf{R}} \mathbf{f}-\bar{r} \mathbf{f}
$$

where $\bar{r}=\mathbf{1}^{\top} \overline{\mathbf{R}} \mathbf{f}=\sum_{j} \sum_{k} \bar{r}^{j k} f^{k}$ is the average growth rate of the total population. Again, the oligomorphic approximation shows that the dynamics of class frequencies is $O(1)$ and solely determined by the morph frequencies and morph means.

\subsection{Dynamics of morph frequencies}

In Appendix A, we show that the dynamics of the within-class morph frequencies $f_{i}^{k}$ can be written as

$$
\frac{\mathrm{d} f_{i}^{j}}{\mathrm{~d} t}=\sum_{k} \frac{f^{k}}{f^{j}}\left(f_{i}^{k} r_{i}^{j k}-f_{i}^{j} \bar{r}^{j k}\right)=\sum_{k} \frac{f^{k}}{f^{j}}\left(f_{i}^{k} r^{j k}\left(\bar{z}_{i}^{k}\right)-f_{i}^{j} \sum_{\ell} f_{\ell}^{k} r^{j k}\left(\bar{z}_{\ell}^{k}\right)\right)+O\left(\varepsilon^{2}\right)
$$

which shows that the morph frequencies $f_{i}^{k}$ also have fast dynamics that depend only on the morph positions and frequencies. Equation (10) is the class-structured extension of the first line of equation (17) in Sasaki \& Dieckmann (2011) and is reminiscent of the replicator equation (Crow \& Kimura, 1970; Ewens, 2004).

It is also useful to introduce the total frequency of morph $i, f_{i}=\sum_{k} f_{i}^{k} f^{k}$, and the vector $\mathbf{u}_{i}$ collecting the morph frequencies $u_{i}^{k}=f_{i}^{k} f^{k} / f_{i}$, which gives the fraction of morph- $i$ individuals which are in class $k$. The dynamics of $\mathbf{u}_{i}$ is then

$$
\frac{\mathrm{d} \mathbf{u}_{i}}{\mathrm{~d} t}=\overline{\mathbf{R}}_{i} \mathbf{u}_{i}-\bar{r}_{i} \mathbf{u}_{i}
$$

where $\overline{\mathbf{R}}_{i}$ is the matrix of morph-specific average rates, $\bar{r}_{i}^{k j}$, and $\bar{r}_{i}=\mathbf{1}^{\top} \overline{\mathbf{R}}_{i} \mathbf{u}_{i}$ is the average growth rate of morph $i$. Hence, equation (11) is the morph-specific version of equation (9). Note the difference between the two morph frequencies $f_{i}^{k}$ and $u_{i}^{k}$. The frequency $f_{i}^{k}$ gives the fraction of morph- $i$ individuals among all class- $k$ individuals, while the frequency $u_{i}^{k}$ is the fraction of class- $k$ individuals among all morph- $i$ individuals. 


\subsection{Dynamics of morph reproductive values}

As in Lion (2018a), equation (11) has a "companion" equation (or adjoint equation, in mathematical terms), which gives the dynamics of the vector of individual reproductive values for morph $i, \mathbf{v}_{i}$

$$
\frac{\mathrm{d} \mathbf{v}_{i}^{\top}}{\mathrm{d} t}=-\mathbf{v}_{i}^{\top} \overline{\mathbf{R}}_{i}+\bar{r}_{i} \mathbf{v}_{i}^{\top}
$$

The reproductive value $v_{i}^{k}(t)$ measures the relative contribution to the future of a morph- $i$ individual in class $k$ at time $t$, and therefore gives an instantaneous measure of the relative quality of class $k$ from the point of view of morph $i$. Note that $\mathbf{v}_{i}$ and $\mathbf{u}_{i}$ are co-normalised such that $\mathbf{v}_{i}^{\top} \mathbf{u}_{i}=1$. This co-normalisation condition means that the average quality of a morph- $i$ individual is 1 at all times (Lion \& Gandon, 2021).

\subsection{The net effect of selection on morph $i$}

Equations (10) tell us whether a given morph increases or decreases in frequency within each class. But suppose we want to know whether the overall effect of selection on morph $i$ is positive or negative. Intuitively, we need to calculate an average frequency over all classes, but how whould this average be calculated? We can do this by weighting each class by its reproductive value, which amounts to expressing fitness effects in each class into a common currency (Fisher, 1930; Taylor, 1990; Lehmann \& Rousset, 2014; Gardner, 2015; Grafen, 2015; Lion, 2018a). The net effect of selection on morph $i$ at a given time can then be captured by calculating the dynamics of this weighted average frequency (Lion, 2018a). In the next sections, we will extend this idea to derive the net effect of selection on the moments of the morph distributions, and show that this accurately describes the eco-evolutionary dynamics when the morph distributions are sufficiently narrow.

\section{Dynamics of morph means}

On the fast time scale where morph means and variances do not change much, equations (8)-(10) are sufficient to describe the eco-evolutionary dynamics of the population. We now look at the longer time scale where morph means $\bar{z}_{i}^{k}$ change slowly but morph variances can be assumed to be fixed, leading to equations that are reminiscent of classical quantitative genetics.

\subsection{Class-specific morph means}

In Appendix B, we show that the dynamics of morph means take the form of a class-structured Price equation (similar to those derived in Lion $(2018 \mathrm{a}, \mathrm{b})$ ). With the oligomorphic approximation, and assuming that the morph distributions are and remain symmetric, we obtain

$$
\frac{\mathrm{d} \bar{z}_{i}^{k}}{\mathrm{~d} t}=\sum_{j} \frac{u_{i}^{j}}{u_{i}^{k}}\left[\left(\bar{z}_{i}^{j}-\bar{z}_{i}^{k}\right) r^{k j}\left(\bar{z}_{i}^{j}\right)+\left.V_{i}^{j} \frac{\partial r^{k j}}{\partial z}\right|_{z=\bar{z}_{i}^{j}}\right]+O\left(\varepsilon^{3}\right)
$$

where the first term depends on the between-class phenotypic differentiation of morph $i$, while the second term is scaled by the within-class morph variance $V_{i}^{j}$. These two terms are $O(\varepsilon)$ and $O\left(\varepsilon^{2}\right)$ respectively, so that the $\bar{z}_{i}^{k}$ 's are slow variables compared to the morph frequencies $f_{i}^{k}$ 's, and the class densities $n^{k}$. In a quasi-equilibrium approximation, this means that, while the $\bar{z}_{i}^{k}$ change slowly, the fast variables immediately track this change so that the right-hand sides of equations (8)-(10), which all explicitly depend on the morph means $\bar{z}_{i}^{k}$, can be set to zero.

A biological interpretation of equation (13) can be obtained by noting that the second term between brackets in equation (13) corresponds to the effect of selection on morph $i$ within class $j$, and depends on the variance $V_{i}^{j}$ of morph $i$ in class $j$ and on the marginal effect of the trait on the vital rates, evaluated at the morph mean in class $j$. On the other hand, the first term between brackets represents the effect of between-class processes: for a given level of phenotypic differentiation $\bar{z}_{i}^{j}-\bar{z}_{i}^{k}$, generated by selection or other processes, demographic transitions from class $j$ to class $k$, which occur at rate 
$r^{k j}\left(\bar{z}_{i}^{k}\right)$, may also contribute to the dynamics of the morph mean $\bar{z}_{i}^{k}$. Finally, the ratio $u_{i}^{j} / u_{i}^{k}$ gives the relative abundance of class $j$ and $k$ in the population of morph- $i$ individuals and is used as a weight to obtain the net change of the morph mean in class $k$, so that classes with a low frequency in the population do not contribute much. Note that, in the absence of class structure, the first term vanishes and we recover equation (25) in Sasaki \& Dieckmann (2011).

\subsection{RV-weighted trait distributions}

Suppose now we are interested in how the different morphs behave on average. How can we calculate the net effect of selection on the morph moments? As we discussed above, we use reproductive values to define a weighted trait distribution (Lion, 2018a)

$$
\widetilde{\phi}_{i}(z, t)=\sum_{k} c_{i}^{k}(t) \phi_{i}^{k}(z, t)
$$

where $c_{i}^{k}(t)=v_{i}^{k}(t) u_{i}^{k}(t)$ is the class reproductive value of morph $i$ in class $k$ at time $t$. Equation (14) weighs the class-specific morph distribution by both the quantity $u_{i}^{k}(t)$ and quality $v_{i}^{k}(t)$ of class- $k$ individuals within the morph- $i$ subpopulation. As intuitively expected, the quality and quantity of a class may change over time, so these are dynamical variables (Lion, 2018a; Lion \& Gandon, 2021). Figure 1 gives a graphical illustration of the process by which class- and morph-specific distributions can be aggregated to obtain morph-specific RV-weighted distributions.

Biologically, equation (14) gives us an appropriate metric to measure the average effect, across all classes, of selection acting on a given morph. By accounting for the relative qualities of each class, it is possible to get rid of any spurious effect due to intrinsic demographic differences between classes. Mathematically, this means that we project the dynamics of moments on a lower-dimensional space, on which we only need to track the moments of an average morph distribution instead of the moments of class-specific morph distributions. For simplicity, we call this approximation the projection on RV space.

\subsection{Projection on RV space}

We can use the projection on RV space to simplify the dynamics of the morph means and variances. Mathematical details are given in Appendix D, but the key biological insight is that, if morph distributions are sufficiently clustered around the morph mean, the dynamics of the morph moments on the slow time scale will closely track the moments of the reproductive-value-weighted morph distribution.

Equation (12) shows that the dynamics of the morph reproductive values are $O(1)$, so that reproductive values change on the same time scale as class frequencies. On this fast time scale, a quasi-equilibrium approximation yields

$$
\overline{\mathbf{R}}_{i} \mathbf{u}_{i}=\mathbf{v}_{i}^{\top} \overline{\mathbf{R}}_{i}=\mathbf{0}
$$

so that the vectors of class frequencies and reproductive values are respectively the right and left eigenvectors of the matrix $\overline{\mathbf{R}}_{i}$ associated with eigenvalue 0 , where $\overline{\mathbf{R}}_{i}$ has elements $\bar{r}_{i}^{k j}=r^{k j}\left(\bar{z}_{i}\right)+O(\varepsilon)$. This is a multi-morph extension of a standard result from monomorphic theory (Taylor, 1990; Rousset, 2004; Lehmann \& Rousset, 2014; Lion, 2018a,b; Priklopil \& Lehmann, 2020).

\subsection{Dynamics of morph means on RV space}

On the slow time scale, the morph mean across all classes can be approximated, to leading order, by the dynamics as the reproductive-weighted morph mean. This yields

$$
\frac{\mathrm{d} \bar{z}_{i}}{\mathrm{~d} t} \approx \frac{\mathrm{d} \widetilde{z}_{i}}{\mathrm{~d} t}=\left.\sum_{j} V_{i}^{j} \sum_{k} v_{i}^{k} \frac{\partial r^{k j}}{\partial z}\right|_{z=\bar{z}_{i}^{j}} u_{i}^{j}+O\left(\varepsilon^{3}\right) .
$$

Thus, the dynamics of the morph mean is a weighted sum of the marginal effect on between-class transition rates, weighted by (1) the variance $V_{i}^{j}$ of the morph in the class of origin $j,(2)$ the individual 
reproductive value $v_{i}^{k}$ of the morph in the destination class, $k$, and (3) the frequency $u_{i}^{j}=f_{i}^{j} f^{j} / f_{i}$ of class- $j$ invididuals in morph $i$. Equation (16) has the same form as equation (8) in Barfield et al. (2011), but with a subtle difference. Indeed, in Barfield et al. (2011) the direction of selection is given by a gradient in the mean transition rates (e.g. the $\bar{r}^{k j}$ in our formalism), but equation (16) depends on a gradient in the transition rates $r^{k j}$. This is because, in contrast to Barfield et al. (2011), we explicitly allow for frequency-dependent selection (Iwasa et al., 1991; Day, 2005; Lion, 2018c). Thus, equation (16) represents a class-structured extension of Lande (1982)'s univariate theorem to frequency-dependent selection and can be used to describe the dynamics of the modes of non-Gaussian, multimodal distributions.

Figure 2 illustrates the convergence to RV space in a simple two-class model (this is a close-up of the dynamics shown in figure S.3a). The time derivatives of morph means in classes $A$ and $B$ (black lines) quickly relax then closely follow the prediction of equation (16) (gray line), until the eco-evolutionary dynamics stabilise.

Note that, in equation (16), the class frequencies, morph frequencies and reproductive values are all calculated using the zeroth-order terms of the equations (8)-(10). In particular, the vectors $\mathbf{v}_{i}$ and $\mathbf{u}_{i}$ satisfy equation (15).

\subsection{Morph-level closure}

Although equation (16) accurately predicts the dynamics of the mean trait after relaxation of the system, it still depends on the habitat-specific moments $\bar{z}_{i}^{k}$ and $V_{i}^{k}$. However, we can go one step further and effectively reduce the dimension of the system, by approximating habitat-specific means $\bar{z}_{i}^{k}$ and variances $V_{i}^{k}$ by the morph means $\bar{z}_{i}$ and $V_{i}$, which leads to:

$$
\frac{\mathrm{d} \bar{z}_{i}}{\mathrm{~d} t} \approx V_{i} \mathbf{v}_{i}^{\top} \mathbf{S}_{i} \mathbf{u}_{i}+O\left(\varepsilon^{3}\right)
$$

where

$$
\mathbf{S}_{i}=\left.\frac{\partial \mathbf{R}}{\partial z}\right|_{z=\bar{z}_{i}}
$$

is the directional selection matrix. The effect of selection on the morph mean is thus scaled by the morph variance, $V_{i}$, and by the selection gradient $\mathbf{v}_{i}^{\top} \mathbf{S}_{i} \mathbf{u}_{i}$, which depends on (1) the marginal effect of a change in the trait on the between-class transition rates $r^{k j}(z)$, evaluated at the morph mean, (2) the relative quality of class $k$ for morph- $i$ individuals, measured by the reproductive value $v_{i}^{k}$, and (3) the relative quantity $u_{i}^{k}$ of class- $k$ individuals among morph- $i$ individuals. This is the multi-morph extension of the classical expression for the class-structured selection gradient (Taylor, 1990; Rousset, 1999, 2004; Lehmann \& Rousset, 2014; Lion, 2018a,b; Priklopil \& Lehmann, 2020), which can be recovered by noting that, in the single-morph case, $f_{i}^{k}=f_{i}=1$, so that $\mathbf{u}_{i}=\mathbf{f}$ and $\mathbf{v}_{i}=\mathbf{v}$.

\section{Dynamics of morph variances}

A classical quantitative genetics approach would typically focus on equations (16) and (17) under the assumption of constant variances. However, this is not sufficient to understand how disruptive selection may shape multi-morph trait distributions, and for this we need to turn to the dynamics of the morph variances. As for the means, we first calculate the dynamics of the class-specific morph variances, before using the variances of the RV-weighted morph distributions to obtain a simpler result. 


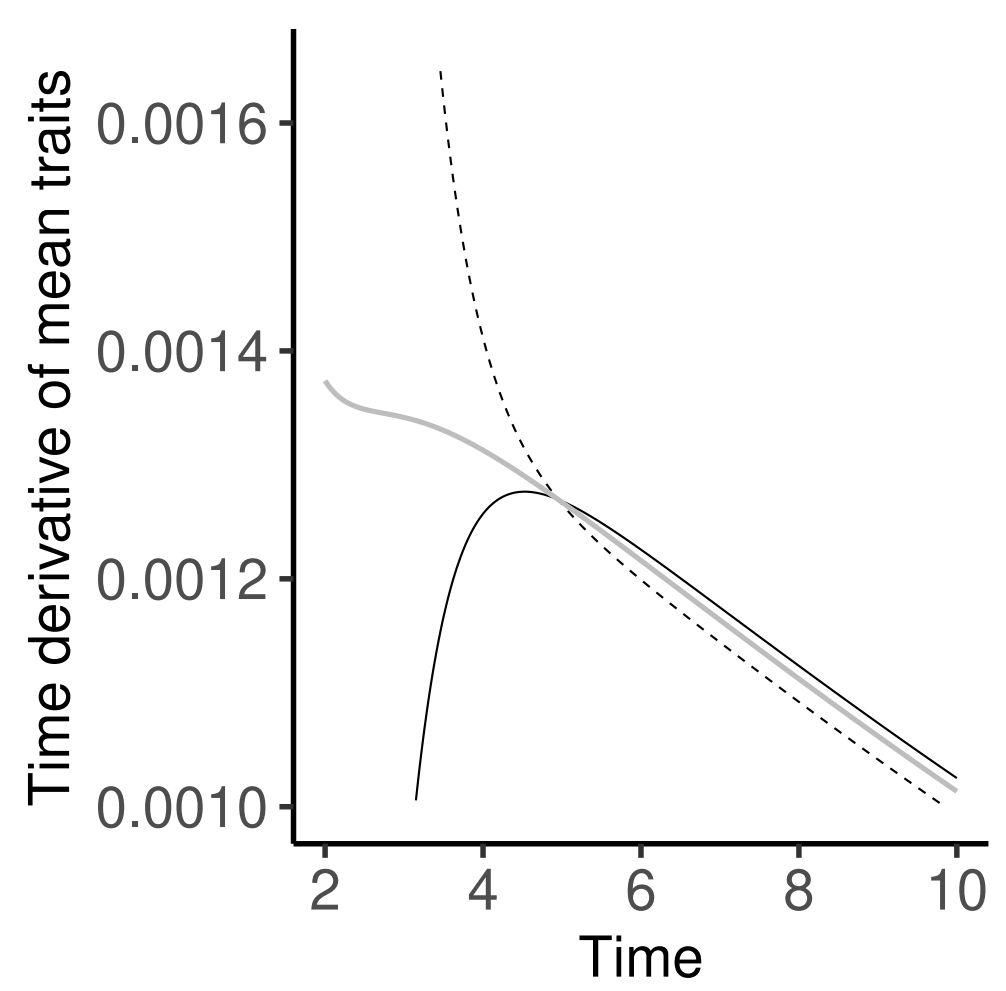

Figure 2: Illustration of the relaxation to RV space in a two-class model. The simulation is the same as in figure S.3a, to which the reader is referred for additional details. The time derivatives $\mathrm{d} \bar{z}_{A, 1} / \mathrm{d} t$ and $\mathrm{d} \bar{z}_{B, 1} / \mathrm{d} t$ are plotted (solid and dashed black lines respectively) and shown to converge towards the prediction of the right-hand side of equation (16) (grey line).

\subsection{Class-specific morph variances}

In Appendix B, we show that the dynamics of the class-specific morph variances can be written as:

$$
\begin{aligned}
\frac{\mathrm{d} V_{i}^{k}}{\mathrm{~d} t}=\sum_{j} \frac{u_{i}^{j}}{u_{i}^{k}}\left[\left(V_{i}^{j}\right.\right. & \left.-V_{i}^{k}+\left(\bar{z}_{i}^{j}-\bar{z}_{i}^{k}\right)^{2}\right) r^{k j}\left(\bar{z}_{i}^{j}\right) \\
& +\left.2\left(\bar{z}_{i}^{j}-\bar{z}_{i}^{k}\right) V_{i}^{j} \frac{\partial r^{k j}}{\partial z}\right|_{z=\bar{z}_{i}^{j}} \\
& \left.+\left.\frac{1}{2}\left(Q_{i}^{j}+\left(\bar{z}_{i}^{j}-\bar{z}_{i}^{k}\right)^{2} V_{i}^{j}-V_{i}^{j} V_{i}^{k}\right) \frac{\partial^{2} r^{k j}}{\partial z^{2}}\right|_{z=\bar{z}_{i}^{j}}\right]+O\left(\varepsilon^{5}\right)
\end{aligned}
$$

The terms on the first, second and third lines are respectively $O\left(\varepsilon^{2}\right), O\left(\varepsilon^{3}\right)$ and $O\left(\varepsilon^{4}\right)$. The term on the first line corresponds to the effect of demographic transitions between classes on variance. Basically it tells us that, even in the absence of selection, changes in the morph variance in class $k$ can be observed if the morph distributions are different across classes (e.g. if the morph mean and variance in class $j$ differ from class $k$ ). The term on the second line represents the effect of directional selection on the morph variance in class $k$, which will be greater when there is substantial phenotypic differentiation between the focal class and the other classes. Finally, the term on the third line represents the effect of disruptive selection on the morph variance, and depends on the fourth moments $Q_{i}^{j}$ and on the morph means and variances. Note that, in the absence of class structure, the first two lines vanish and we recover equation (33) in Sasaki \& Dieckmann (2011). 


\subsection{Dynamics of morph variances on RV space}

As for the morph mean we can use reproductive values to derive an aggregate equation for the dynamics of the morph variance, $V_{i}$. In its most compact form, it takes the form of a Price equation

$$
\frac{\mathrm{d} V_{i}}{\mathrm{~d} t} \approx \frac{\mathrm{d} \widetilde{V}_{i}}{\mathrm{~d} t}=\sum_{j} \sum_{k} v_{i}^{k} \operatorname{Cov}_{\phi_{i}^{j}}\left(\left(z-\widetilde{z}_{i}\right)^{2}, r^{k j}(z)\right) u_{i}^{j} .
$$

where the covariance is calculated over the distribution $\phi_{i}^{j}(z, t)$. The oligomorphic approximation allows us to further expand the covariance (see equation (20) in Appendix D) as

$$
\frac{\mathrm{d} V_{i}}{\mathrm{~d} t} \approx 2\left(V_{i}\right)^{2}\left[\mathbf{v}_{i}^{\top} \mathbf{F}_{i} \mathbf{u}_{i}+\mathbf{v}_{i}^{\top} \mathbf{S}_{i}\left(\mathbf{d}_{i} \circ \mathbf{u}_{i}\right)\right]+O\left(\varepsilon^{5}\right) .
$$

where the notation $\circ$ denotes the elementwise (Hadamard) product. The first-term between brackets depends on the matrix

$$
\mathbf{F}_{i}=\left.\frac{1}{2} \frac{\partial^{2} \mathbf{R}}{\partial z^{2}}\right|_{z=\bar{z}_{i}}
$$

It is the class-structured analog of equation (37) in Sasaki \& Dieckmann (2011) and gives the net effect of the curvature of the fitness functions $r^{k j}(z)$ on the dynamics of variance.

The second term between brackets depends on the directional selection matrix $\mathbf{S}_{i}$, and on the vector $\mathbf{d}_{i}$ of phenotypic differentiations $\left(\bar{z}_{i}^{j}-\widetilde{z}_{i}\right) / V_{i}$. The operation $\mathbf{d}_{i} \circ \mathbf{u}_{i}$ returns a vector with elements $u_{i}^{j}\left(\bar{z}_{i}^{j}-\widetilde{z}_{i}\right) / V_{i}(1 \leq j \leq K)$. This second term represents the additional effect of directional selection on the dynamics of morph variance. Intuitively, these two effects can be understood from the decomposition of the morph variance into class-specific morph moments:

$$
\widetilde{V}_{i}=\sum_{k} c_{i}^{k} V_{i}^{k}+\sum_{k} c_{i}^{k}\left[\left(\bar{z}_{i}^{k}\right)^{2}-\left(\widetilde{z}_{i}\right)^{2}\right]
$$

The first term shows that, when the class means are equal, the morph variance $\widetilde{V}_{i}$ is the weighted average of the class-specific morph variances $V_{i}^{k}$. The second term shows that, even when the class variances $V_{i}^{k}$ are zero, substantial differentiation between classes $\left(\bar{z}_{i}^{k} \neq \widetilde{z}_{i}\right)$ can contribute to morph variance. Hence, changes in the mean traits have a direct effect on the dynamics of the variance, which is proportional to the strength of selection in each class (captured by the matrix $\mathbf{S}_{i}$ ) and the level of phenotypic differentiation in each class, compared to the mean morph value (captured by the vector $\left.\mathbf{d}_{i}\right)$.

Note that the second term between brackets in equation (20) still depends on the class-specific morph means, $\bar{z}_{i}^{j}$. On the RV space, the phenotypic differentiation will typically have a quasiequilibrium value (Lion, 2018b) which we can express solely in terms of $\mathbf{v}_{i}, \mathbf{u}_{i}, \overline{\mathbf{R}}\left(\bar{z}_{i}\right)$ and $\mathbf{S}_{i}$, leading to a closed system at the morph level. In the next section, we provide such an expression in a two-class model.

\subsection{The two-class model}

This general framework can be applied to the special, but important case of two-class models to gain further insights on how stabilising and disruptive selection are affected by the class frequencies and reproductive values.

Consider a population structured in two classes $A$ and $B$. As shown in the general case, the dynamics of morph means and variances are given by the following system of equations:

$$
\begin{aligned}
\frac{\mathrm{d} \bar{z}_{i}}{\mathrm{~d} t} & =V_{i} \mathbf{v}_{i}^{\top} \mathbf{S}_{i} \mathbf{u}_{i} \\
\frac{\mathrm{d} V_{i}}{\mathrm{~d} t} & =2 V_{i}^{2}\left[\mathbf{v}_{i}^{\top} \mathbf{F}_{i} \mathbf{u}_{i}+\mathcal{D}_{i}\right]
\end{aligned}
$$


where $\mathcal{D}_{i}$ gives the effect of directional selection on the variance dynamics. For the two-class model, this effect can be calculated as

$$
\mathcal{D}_{i}=\frac{\left(c_{i}^{A} c_{i}^{B}\right)^{3 / 2}}{\sqrt{r^{A B}\left(\bar{z}_{i}\right) r^{B A}\left(\bar{z}_{i}\right)}}\left(\left.\frac{\partial r^{A A}}{\partial z}\right|_{\bar{z}_{i}}-\left.\frac{\partial r^{B B}}{\partial z}\right|_{\bar{z}_{i}}\right)\left(\left.\frac{\partial r^{A A}}{\partial z}\right|_{\bar{z}_{i}}+\left.\frac{v_{i}^{B}}{v_{i}^{A}} \frac{\partial r^{B A}}{\partial z}\right|_{\bar{z}_{i}}-\left.\frac{\partial r^{B B}}{\partial z}\right|_{\bar{z}_{i}}-\left.\frac{v_{i}^{A}}{v_{i}^{B}} \frac{\partial r^{A B}}{\partial z}\right|_{\bar{z}_{i}}\right)
$$

Interestingly, equation (22) shows that directional selection will have a significant effect on the dynamics of variance if three conditions are met: (1) there is enough differentiation between the two classes, as measured by the product of class reproductive values $c_{i}^{A} c_{i}^{B},(2)$ the slopes of the functions $r^{A A}$ and $r^{B B}$ at the morph means are sufficiently different, and (3) the marginal reproductive outputs of $A$ and $B$ individuals are sufficiently different. The latter condition is satisfied when the second bracketed term is non-zero. Note that the ratios $v_{i}^{B} / v_{i}^{A}$ and $v_{i}^{A} / v_{i}^{B}$ can be interpreted as conversion factors to evaluate the $A$ and $B$ descendants in the same currency.

In a two class-model, the class-reproductive values satisfy the following useful quasi-equilibrium relationship:

$$
c_{i}^{B}=v_{i}^{B} u_{i}^{B}=\frac{r^{A B}\left(\bar{z}_{i}\right)\left(u_{i}^{B}\right)^{2}}{r^{B A}\left(\bar{z}_{i}\right)\left(u_{i}^{A}\right)^{2}+r^{A B}\left(\bar{z}_{i}\right)\left(u_{i}^{B}\right)^{2}}=1-c_{i}^{A} .
$$

In the next section, we show how equations (21)-(23) can be used to analyse a specific model of local adaptation in two-habitat migration-selection models.

\subsection{Moment closure}

As typical of moment methods, the dynamics of morph variances (18) depend on higher-order moments, notably the $Q_{i}^{j}$,s, which are the fourth central moments of the morph distribution in class $j$, i.e. $Q_{i}^{j}=\int\left(z-\bar{z}_{i}^{j}\right)^{4} \phi_{i}^{j}(z, t) \mathrm{d} z$. Hence, we need to close the system using a suitable moment closure approximation. For unstructured populations, Sasaki \& Dieckmann (2011) studied two moment closure approximations, namely the Gaussian approximation and the house-of-cards approximation. In this paper, we focus on the Gaussian approximation, and therefore assume that the morph distributions $\phi_{i}^{k}(z)$ are normal. Biologically, this means that the total distribution of the trait can be viewed as a weighted sum of normal distributions. Mathematically, this entails that $Q_{i}^{j}=3\left(V_{i}^{j}\right)^{2}$, which is sufficient to close the system.

Together, equations (8), (9), (13) and (18) form the full oligomorphic approximation of the ecoevolutionary dynamics. It can be used for instance to investigate how an initially unimodal distribution may split into different modes as a consequece of class structure and frequency-dependent selection. For reference, a summary of these equations is given in Box 1, and a summary of the equations using the projection on RV space is given in Box 2.

\section{Example 1: resource competition model}

As an illustration of the method, we consider a population inhabiting two habitats, $A$ and $B$, coupled by migration and characterised by different fitness functions. We define $m_{j k}$ as the migration rate from $k$ to $j$ and $\rho_{k}(z)$ as the growth rate of individuals in habitat $k$, which is a function of a focal trait $z$ measuring competitive ability. With our notations, we have

$$
\begin{aligned}
& r^{A A}(z)=\rho_{A}(z)-m_{B A}, \\
& r^{A B}(z)=m_{A B}, \\
& r^{B A}(z)=m_{B A}, \\
& r^{B B}(z)=\rho_{B}(z)-m_{A B} .
\end{aligned}
$$

Within each habitat, competition between individuals depends on the competitive ability $z$. We further assume that there is a quadratic cost to competitiveness and that each habitat is characterised 
by a value $\theta_{k}$ which minimises the cost. We thus write

$$
\rho_{k}(z)=b-g\left(z-\theta_{k}\right)^{2}-n^{k} \int a(z-y) \phi^{k}(y) \mathrm{d} y
$$

where $b$ is the birth rate, $g$ is the fecundity cost, $\theta_{k}$ is the optimum in habitat $k$, and the competition kernel $a(z-y)$ represents the effect of competition by an individual with trait $y$ on a an individual with trait $z$.

Importantly, the fitness functions $\rho_{k}$ depend on the distributions $\phi^{k}(y, t)$. Following Sasaki \& Dieckmann (2011), we decompose this distribution into a sum of the distributions $\phi_{\ell}(y, t)$ and, for each of these distributions, we use a Taylor expansion of the competition kernel near $y=\bar{z}_{\ell}^{k}$ to express the competition experienced by a focal individual in terms of the means of all the other morphs. We obtain (Appendix S.3)

$$
r^{A A}(z)=b-g\left(z-\theta_{A}\right)^{2}-n^{A} \sum_{\ell} f_{\ell}^{A} a\left(z-\bar{z}_{\ell}^{A}\right)-m_{B A}+O\left(\varepsilon^{2}\right)
$$

and a similar expression for habitat $B$. We can use this expression to calculate the quantities $r^{k j}\left(\bar{z}_{i}\right)$, and the partial derivatives evaluated at $z=\bar{z}_{i}$, and plug these expressions in the equations of Box 1 to obtain the final oligomorphic approximation for this model.

A full analysis of the model is beyond the scope of this paper, but we show in figure 3 the oligomorphic dynamics for a specific choice of parameters. Figure 3c shows that, starting from an effectively monomorphic population (where the two morphs have the same mean values), disruptive selection leads to the splitting of the population into a bimodal distribution after $t \approx 1000$. Disruptive selection is indicated by the explosion of morph variances at the same time. The population then stabilises around a dimorphic equlibrium distribution with means $\bar{z}_{1}^{*}$ and $\bar{z}_{2}^{*}$. Stabilising selection is indicated by the decrease in variance after branching. Figure $3 \mathrm{~b}$ further shows that the morphs have different frequencies in each habitat (i.e. morph 1 is slightly more abundant in habitat $B$ while morph 2 is slightly more abundant in habitat $A$ ), but tend to have similar values for the means and variances in both habitats. This observation suggests that it may be interesting to find a simplified description of the population at the morph level, by aggregating habitat-specific morph moments into a single measure. This is the goal of the RV projection, and we will show its usefulness in the next section.

\section{Example 2: local adaptation in a two-habitat mutation-selection- migration model}

As a proof-of-concept application, we use our approach to investigate how migration and selection interplay to favour or hamper the generation of polymorphism in a population that can exploit two habitats with different qualities. In particular, we show that the notion of habitat quality in polymorphic populations can be captured through the concept of morph-specific reproductive values we introduced in the previous section.

\subsection{A mutation-selection-migration model}

As in our previous example, we consider a population inhabiting two habitats, $A$ and $B$, coupled by migration and characterised by different fitness functions, but we assume that competition is independent of the focal trait. Our model is effectively the same as the one proposed by Meszéna et al. (1997), Ronce \& Kirkpatrick (2001), Débarre et al. (2013), and Mirrahimi \& Gandon (2020), with

$$
\rho_{k}(z)=b-n^{k}-g\left(z-\theta_{k}\right)^{2} .
$$

In contrast with most of these previous studies, we consider the possibility of asymmetric migration rates $\left(m_{A B} \neq m_{B A}\right)$.

In addition to the effects of selection, we also consider the effect of mutation on the eco-evolutionary dynamics. We do so by assuming that mutations occur at rate $\mu$ and that the mutation effects follow a distribution $M$ with mean 0 (mutation has no directional effect) and variance $\sigma_{M}^{2}$. Under these 


\section{(a) Densities}

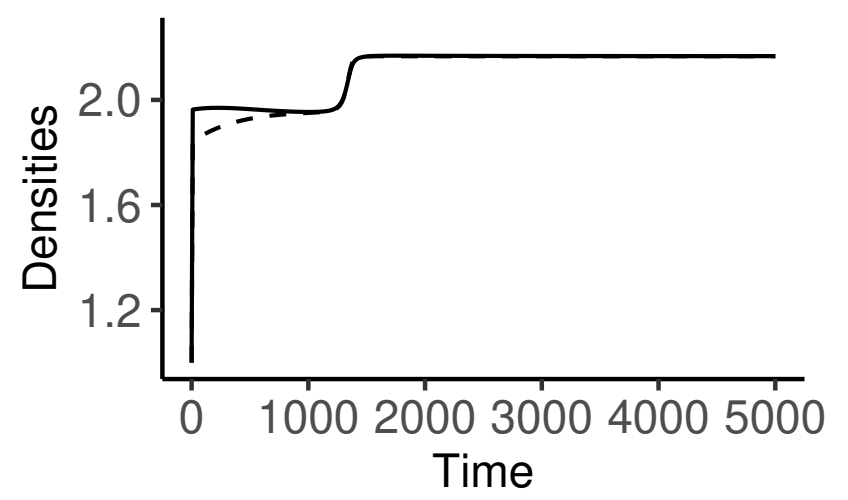

(b) Morph frequencies

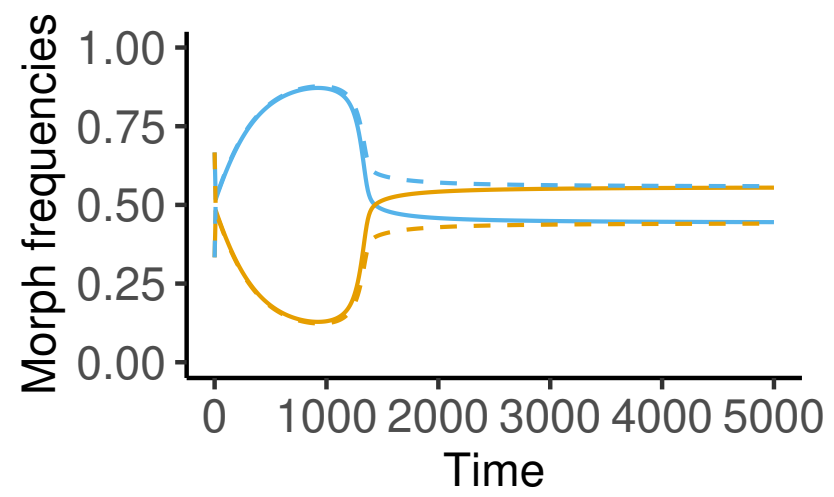

(c) Morph means

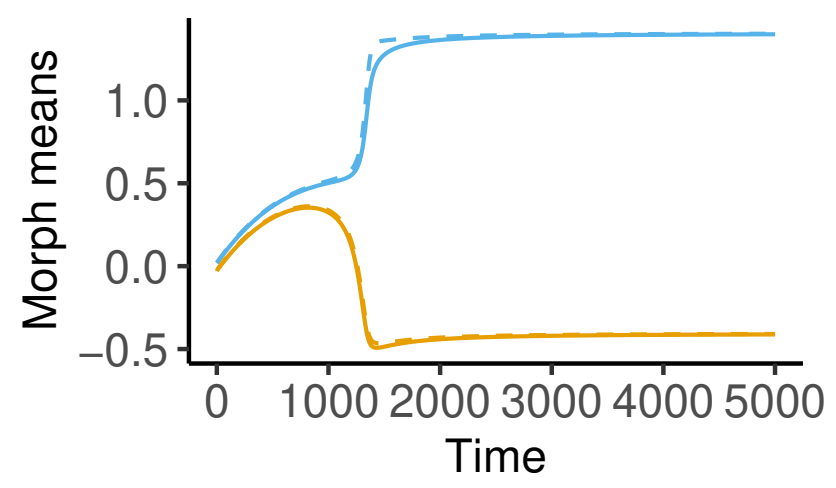

(d) Morph variances

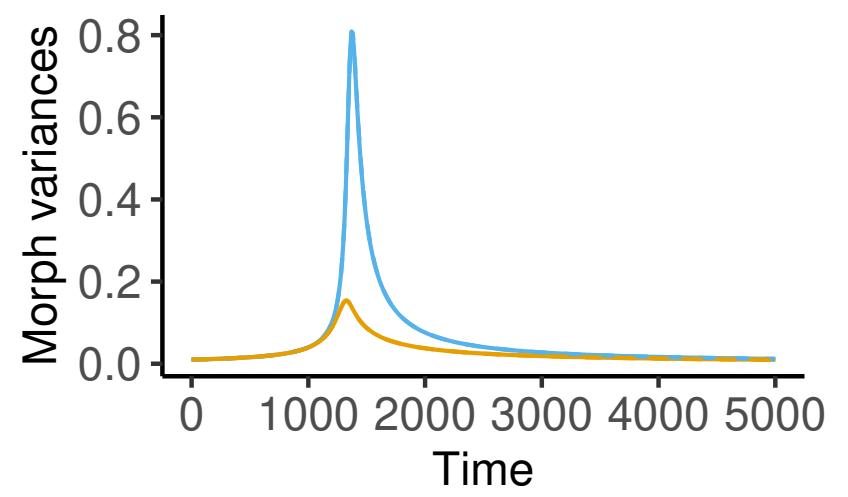

Figure 3: Oligomorphic dynamics of the resource competition model. (a) Dynamics of densities of individuals $n^{k}$ in habitat $A$ (solid line) and $B$ (dashed line). (b) Dynamics of the morph frequencies $f_{i}^{k}$ in habitat $A$ (solid line) and $B$ (dashed lines) for morph 1 (blue) and 2 (orange). (c) Dynamics of the morph means $\bar{z}_{i}^{k}$. (d) Dynamics of the morph variances $V_{i}^{k}$. Parameters: $b=1, g=0.1$, $\theta_{A}=0=1-\theta_{B}, m_{A B}=m_{B A}=0.3, a(x)=0.5 \exp \left(-x^{2} / 8\right)$. 


\section{Box 1: Class-structured oligomorphic dynamics}

To fix ideas, we collect in this box the full system of equations obtained under the oligomorphic approximation. The $(3 M+1) K$ variables are the class densities $n^{k}$, the morph frequencies $f_{i}^{k}$, the morph means $\bar{z}_{i}^{k}$ and the morph variances $V_{i}^{k}(1 \leq i \leq M, 1 \leq k \leq K)$. We have

$$
\begin{aligned}
& \frac{\mathrm{d} n^{k}}{\mathrm{~d} t}=\sum_{j} \sum_{i} r_{i}^{k j}\left(\bar{z}_{i}^{j}\right) f_{i}^{j} n^{j} \\
& \frac{\mathrm{d} f_{i}^{k}}{\mathrm{~d} t}=\sum_{j} \frac{f^{j}}{f^{k}}\left(f_{i}^{j} r^{k j}\left(\bar{z}_{i}^{k}\right)-f_{i}^{k} \sum_{\ell} f_{\ell}^{j} r^{k j}\left(\bar{z}_{\ell}^{j}\right)\right) \\
& \frac{\mathrm{d} \bar{z}_{i}^{k}}{\mathrm{~d} t}=\sum_{j} \frac{u_{i}^{j}}{u_{i}^{k}}\left[\left(\bar{z}_{i}^{j}-\bar{z}_{i}^{k}\right) r^{k j}\left(\bar{z}_{i}^{j}\right)+\left.V_{i}^{j} \frac{\partial r^{k j}}{\partial z}\right|_{\bar{z}_{i}^{j}}\right] \\
& \frac{\mathrm{d} V_{i}^{k}}{\mathrm{~d} t}=\sum_{j} \frac{u_{i}^{j}}{u_{i}^{k}}\left[\left(V_{i}^{j}-V_{i}^{k}+\left(\bar{z}_{i}^{j}-\bar{z}_{i}^{k}\right)^{2}\right) r^{k j}\left(\bar{z}_{i}^{j}\right)+\left.2\left(\bar{z}_{i}^{j}-\bar{z}_{i}^{k}\right) V_{i}^{j} \frac{\partial r^{k j}}{\partial z}\right|_{\bar{z}_{i}^{j}}\right. \\
&\left.\quad+\left.\left(Q_{i}^{j}+\left(\bar{z}_{i}^{j}-\bar{z}_{i}^{k}\right)^{2} V_{i}^{j}-V_{i}^{j} V_{i}^{k}\right) \frac{\partial^{2} r^{k j}}{\partial z^{2}}\right|_{\bar{z}_{i}^{j}}\right]
\end{aligned}
$$

with $f^{j}=n^{j} / \sum_{k} n^{k}$ the fraction of individuals in class $j$ and $u_{i}^{j}=f_{i}^{j} f^{j} / \sum_{k} f_{i}^{k} f^{k}$ the fraction of morph- $i$ individuals in class $j$. The system can be closed using a moment closure approximation such as the Gaussian closure $\left(Q_{i}^{j}=3\left(V_{i}^{j}\right)^{2}\right)$, and numerically integrated.

It is important to note that all the equations are coupled. In particular, the dynamics of the means of morph $i$ may depend on the means and frequcncies of the other morphs. For instance, in the resource competition model described in the main text, we have:

$$
\left.\frac{\partial r^{A A}}{\partial z}\right|_{\bar{z}_{i}}=-2 g\left(\bar{z}_{i}-\theta_{A}\right)-n^{A} \sum_{\ell} f_{\ell}^{A} a^{\prime}\left(\bar{z}_{i}^{A}-\bar{z}_{\ell}^{A}\right)
$$

which shows that the competition experienced by morph $i$ depends on the other morphs through their frequencies $f_{\ell}^{A}$ and mean trait values $\bar{z}_{\ell}^{A}$. 


\section{Box 2: Projection on RV space}

By projecting on RV space, the dynamics of the system in Box 1 can be reduced to a system of $2 M$ equations for the aggregate morph means $\bar{z}_{i}$ and variances $V_{i}(1 \leq i \leq M)$.

On the slow time scale, we have

$$
\begin{aligned}
& \frac{\mathrm{d} \bar{z}_{i}}{\mathrm{~d} t}=V_{i} \mathbf{v}_{i}^{\top} \mathbf{S}_{i} \mathbf{u}_{i} \\
& \frac{\mathrm{d} \bar{V}_{i}}{\mathrm{~d} t}=2\left(V_{i}\right)^{2}\left[\mathbf{v}_{i}^{\top} \mathbf{F}_{i} \mathbf{u}_{i}+\mathcal{D}_{i}\right]
\end{aligned}
$$

where the matrices $\mathbf{S}_{i}$ and $\mathbf{F}_{i}$ have elements $\left.\frac{\partial r^{k j}}{\partial z}\right|_{\bar{z}_{i}}$ and $\left.\frac{\partial^{2} r^{k j}}{\partial z^{2}}\right|_{\bar{z}_{i}}$ respectively, and $\mathcal{D}_{i}$ represents the effect of directional selection on the dynamics of variance.

On the fast time scale, the ecological variables can be calculated from the following quasiequilibrium relationships

$$
\begin{aligned}
& \mathbf{0}=\mathbf{R}\left(\bar{z}_{i}\right) \mathbf{u}_{i}=\mathbf{v}_{i}^{\top} \mathbf{R}\left(\bar{z}_{i}\right) \\
& 0=\sum_{j} \frac{f^{j}}{f^{k}}\left(f_{i}^{j} r^{k j}\left(\bar{z}_{i}\right)-f_{i}^{k} \sum_{\ell} f_{\ell}^{j} r^{k j}\left(\bar{z}_{\ell}\right)\right)
\end{aligned}
$$

assumptions, mutation has no effect on the dynamics of the mean traits, but adds an extra term to the dynamics of the morph variances, which is equal to the mutational variance $V_{M}=\mu \sigma_{M}^{2}$ (Kimura, 1965; Lande, 1975; Sasaki \& Dieckmann, 2011).

Together with the equations in Box 2, these expressions for the vital rates allow us to derive the following equations for the dynamics of morph means and variances (Appendix S.2):

$$
\begin{aligned}
\frac{\mathrm{d} \bar{z}_{i}}{\mathrm{~d} t} & =-2 g V_{i}\left[\bar{z}_{i}-c_{i}^{A} \theta_{A}+c_{i}^{B} \theta_{B}\right] \\
\frac{\mathrm{d} V_{i}}{\mathrm{~d} t} & =-2 g V_{i}^{2}\left[1-\frac{4 g}{m}\left(c_{i}^{A} c_{i}^{B}\right)^{3 / 2}\left(\theta_{B}-\theta_{A}\right)^{2}\right]+V_{M}
\end{aligned}
$$

where $m=\sqrt{m_{A B} m_{B A}}$ is the geometric mean of the dispersal rates, and the morph-specific class reproductive values are given by their quasi-equilibrium expressions (equation (23)):

$$
c_{i}^{B}=1-c_{i}^{A}=\frac{m_{A B}\left(u_{i}^{B}\right)^{2}}{m_{B A}\left(u_{i}^{A}\right)^{2}+m_{A B}\left(u_{i}^{B}\right)^{2}} .
$$

Using these equations, we investigate three main questions: (1) What are the attractors of the ecoevolutionary dynamics? (2) When does selection lead to a unimodal vs. bimodal equilibrium distribution? and (3) What is the effect of habitat-specific mutation on the evolutionary outcome? Since a full analysis of the model would be beyond the reach of this article, we focus on the most salient features in the main text and briefly consider additional technicalities in Appendix S.2.

\subsection{Evolutionary attractors}

From equation (25), we see that the morph means stabilise when

$$
\bar{z}_{i}=c_{i}^{A} \theta_{A}+c_{i}^{B} \theta_{B}
$$

It is important to note that, because of the quasi-equilibrium approximation, the class reproductive values are also functions of $\bar{z}_{i}$, so that equation (28) is only implicit. Nonetheless, the biological implication is that the potential attractors for the dynamics of the morph means correspond to the reproductive-value-weighted mean of the habitat optima. Hence, the outcome of selection for a given 
morph depends on the relative difference in quality between habitats, measured by $c_{i}^{B}-c_{i}^{A}$. When $c_{i}^{B}>c_{i}^{A}$, the morph mean will be biased towards the optimum of habitat $B$, whereas the opposite will be observed for $c_{i}^{B}<c_{i}^{A}$. In the following, we fix $\theta_{A}=0=1-\theta_{B}$, without loss of generality.

The most important result of the model is that, when the mean migration rate is sufficiently high, the eco-evolutionary dynamics settle on an unimodal distribution, while bimodal distributions can be generated if the mean migration rate is below a threshold $m_{c}$ (figure 4a). Simulations of the full model, without the oligomorphic approximation, show that our reproductive-value-weighted oligomorphic approximation can accurately predict both the unimodal and bimodal endpoints (figure $4 a)$.

At a unimodal distribution, the class reproductive values of the different morphs are equal (e.g. $c_{1}^{B}=c_{2}^{B}=c$ if we start with 2 morphs), so there is effectively a single morph in the population with mean trait value $\bar{z}=c$ (figure $4 \mathrm{c}$ ). In contrast, if different morphs have distinct class reproductive values (e.g. $c_{1}^{B} \neq c_{2}^{B}$ ), the eco-evolutionary dynamics converge towards a bimodal equilibrium distribution, where the morphs have different means $\left(\bar{z}_{1}=c_{1}^{B}\right.$ for morph 1 and $\bar{z}_{2}=c_{2}^{B}$ for morph 2), and occur with different frequencies in the two habitats. The trait distributions in each habitat therefore have two peaks, one around $\bar{z}_{1}$ and one around $\bar{z}_{2}$, the heights of the peaks being determined by the morph frequencies $f_{i}^{k}$ and the densities $n_{A}$ and $n_{B}$ (figure $4 \mathrm{~b}$ ). Using our formalism, it is possible to analytically calculate the position of each morph. For $m<g / 2$, we obtain:

$$
\begin{aligned}
& \bar{z}_{1}^{*}=c_{1}^{B}=\frac{1}{2}+\frac{1}{2} \sqrt{1-\frac{4 m^{2}}{g^{2}}} \\
& \bar{z}_{2}^{*}=c_{2}^{B}=\frac{1}{2}-\frac{1}{2} \sqrt{1-\frac{4 m^{2}}{g^{2}}} .
\end{aligned}
$$

This is the same expression as obtained by Débarre et al. (2013) for symmetric migration, except that our expression depends on the mean migration rate $m=\sqrt{m_{A B} m_{B A}}$. Note that, even with asymmetric migration, the equilibrium is symmetric as $\bar{z}_{1}^{*}=1-\bar{z}_{2}^{*}$. For completeness, we note that the oligomorphic analysis predicts bistability when $m<m_{c}$, with some initial conditions leading to unimodal equilibrium distributions (Appendix S.2), although the bimodal distribution appears to be the more robust evolutionary outcome (see also Mirrahimi \& Gandon (2020) for a global stability analysis).

\subsection{Local adaptation}

At the bimodal equilibrium, the two morphs have different frequencies in the two habitats and therefore the habitat-specific distributions are distinct (figure $4 \mathrm{~b}$ ). The level of habitat differentiation in mean traits provides a measure of local adaptation (Débarre et al., 2013) and can be calculated as

$$
D=\bar{z}^{B}-\bar{z}^{A}=\left(f_{1}^{B}-f_{1}^{A}\right)\left(\bar{z}_{1}-\bar{z}_{2}\right),
$$

which shows that, at equilibrium, local adaptation depends (1) on the difference in morph frequencies between the two habitats, and (2) on the difference between the class reproductive values of the two morphs. In our model, this can be calculated as:

$$
D^{*}=1-\frac{1}{g}\left(\frac{f^{B}}{f^{A}} m_{A B}+\frac{f^{A}}{f^{B}} m_{B A}\right)
$$

which simplifies to $D^{*}=1-2 \mathrm{~m} / \mathrm{g}$ for symmetric migration, as found in Débarre et al. (2013). Higher migration thus leads to lower local adaptation.

\subsection{Habitat differentiation and disruptive selection}

When does the interplay between migration and selection leads to unimodal vs bimodal equilibrium trait distributions? To answer this question, we turn to the dynamics of morph variances (equation (26)), which provides a useful interpretation of evolutionarily stability in terms of reproductive values. 
Indeed, $\Delta_{i}=1-4 c_{i}^{A} c_{i}^{B}$ is a measure of differentiation in habitat quality, as measured by reproductive values: it reaches its maximal value, 1 , when $c_{i}^{B}=0$ or $c_{i}^{B}=1$, and is zero when the habitats have equal qualities $\left(c_{i}^{A}=c_{i}^{B}=1 / 2\right)$. In the absence of mutation $\left(V_{M}=0\right)$, selection on morph $i$ is therefore stabilising if habitat differentiation is above a threshold determined by the ratio between migration $(m)$ and selection $(g)$ :

$$
\Delta_{i}>1-\left(\frac{2 m}{g}\right)^{2 / 3}
$$

Note that, for $m>g / 2$, this is always satisfied, but with a lower mean migration rate, stability depends on the magnitude of habitat differentiation. Hence, for $m<g / 2$, disruptive selection is possible and may lead to the splitting of an initially unimodal distribution into two peaks.

Equation (26) further shows that the dimorphic attractor $\left(\bar{z}_{1}^{*}, \bar{z}_{2}^{*}\right)$ is evolutionarily stable when $m<g / 2$ and unstable otherwise. However, there is a critical value $m_{c}<g / 2$ above which the bimodal equilibrium loses its demographic stability and one of the two morphs goes extinct (figure S.5a). Hence, for $m>m_{c}$, the only possible evolutionary outcome is a unimodal distribution. For $m<m_{c}$, multistability is possible, where the system can converge towards unimodal or bimodal distributions depending on initial conditions (figure 4a).

\subsection{Mutation-selection-migration balance}

With mutation and stabilising selection, the morph variances can settle at a mutation-selection equilibrium. From equation (26), the equilibrium variance is then given by

$$
V_{i}^{*}=\sqrt{\frac{V_{M}}{2 g\left[1-\frac{4 g}{m}\left(c_{i}^{A} c_{i}^{B}\right)^{3 / 2}\right]}}
$$

The numerator is the effect of mutation on variance, while the denominator gives the effect of selection. When only one habitat is present (e.g. $c_{i}^{A}=1$ and $c_{i}^{B}=0$ ), the morph variance at equilibrium is $\sqrt{V_{M} / 2 g}$, which is equation (17) in Débarre et al. (2013). For the bimodal attractor, both morphs have the same equilibrium variance:

$$
V_{1}^{*}=V_{2}^{*}=\sqrt{\frac{V_{M}}{2 g\left(1-\frac{4 m^{2}}{g^{2}}\right)}}
$$

It is straightforward to generalise this result to the case where the two habitats have different mutation rates, leading to distinct habitat-specific mutational variances $V_{M}^{A}$ and $V_{M}^{B}$. Then, $V_{M}=c_{i}^{A} V_{M}^{A}+$ $c_{i}^{B} V_{M}^{B}$ in equation (33), so that the effect of mutation takes the form of a reproductive-value-weighted average mutational variance. In this case, each morph has a different equilibrium variance, which can be analytically calculated using equation (29). Figure 5a shows that this accurately predicts the equilibrium values of morph variances under mutation-selection balance.

As mutation increases, the accurary of the oligomorphic approximation is expected to decrease, as high mutation will tend to generate broader morph distributions. Nonetheless, figure 5b shows that our analytical prediction of the differentiation $D=\bar{z}^{B}-\bar{z}^{A}$ remains good even for relatively large values of the mutation variance $V_{M}$ (see Appendix S.2 for a more detailed discussion).

\section{Discussion}

We have developed a novel theoretical framework to model the eco-evolutionary dynamics of polymorphic, class-structured populations. Our analysis makes two key contributions. First, we extend the recently developed oligomorphic approximation (Sasaki \& Dieckmann, 2011) to class-structured populations. Since class structure is a major feature of natural biological populations, this allows the method to be applied to a broad range of ecological scenarios, taking into account individual differences in state including age, spatial location, infection or physiological status, and species. Second, we combine the oligomorphic approximation with recent theory on reproductive values to obtain a 
(a) Bimodal vs unimodal distributions

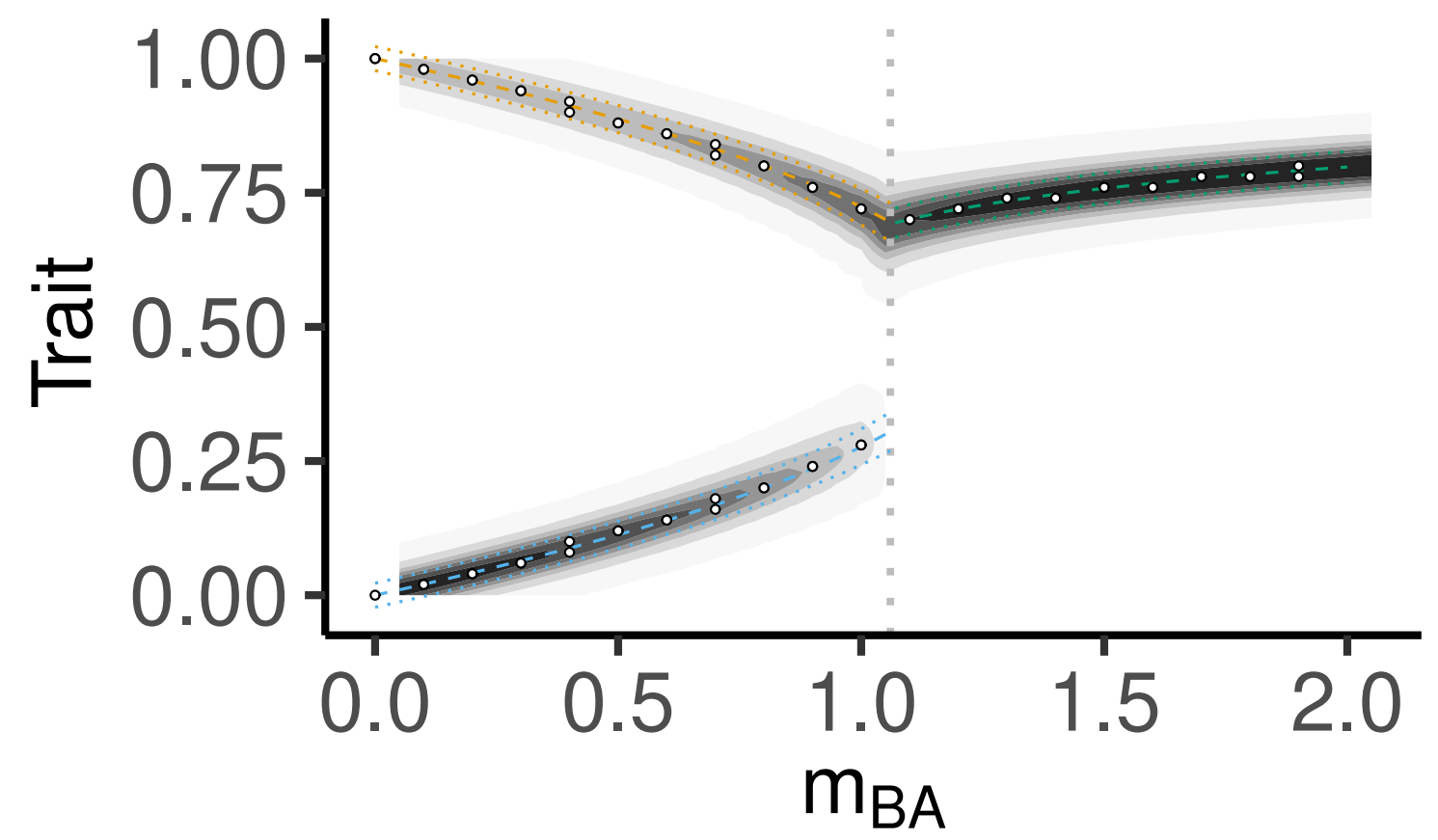

(b) Low $m$ - Bimodal distribution
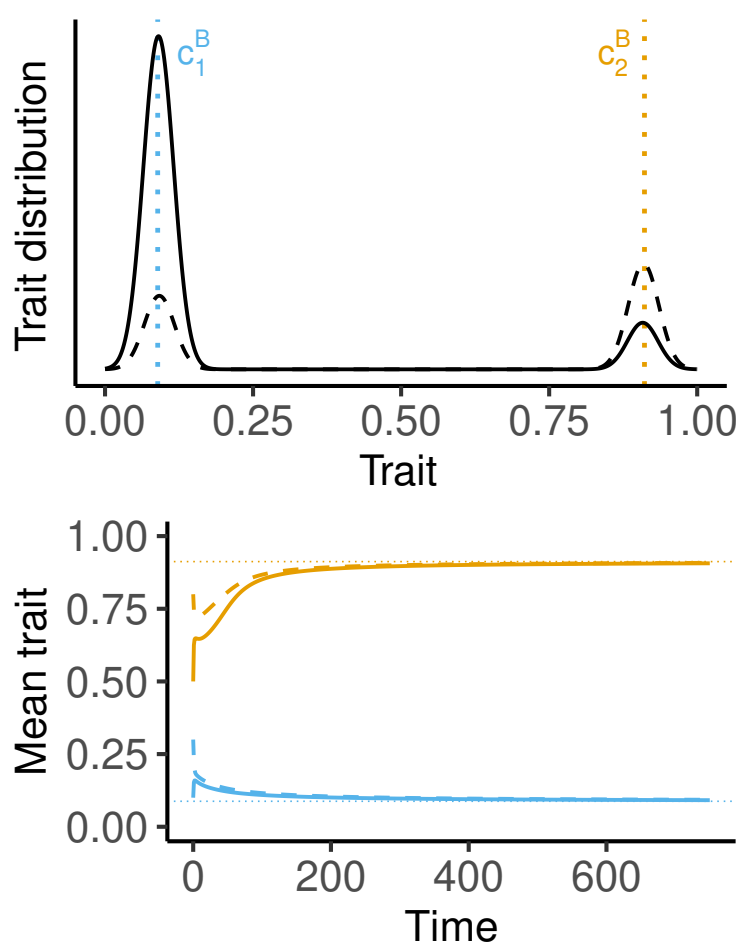

(c) High $m$ - Unimodal distribution
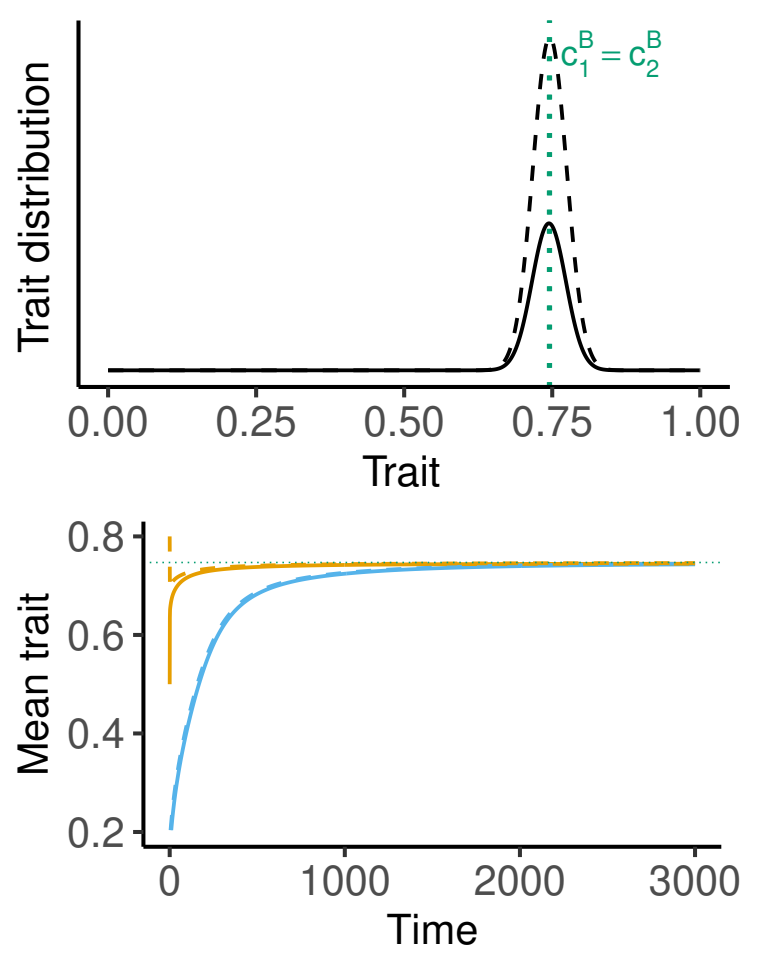

Figure 4: Bimodal vs. unimodal equilibrium distributions. Figure (a) shows how the equilibrium distribution changes when $m_{A B}=0.8$ is fixed and $m_{B A}$ varies. For simplicity, we only show the dimorphic solution in the bistability region. The vertical dotted line represents the value $m_{B A} \approx 1.06$ at which the dimorphic equilibrium loses its demographic stability and one of the two morphs goes extinct. The analytical predictions using the projection on RV space are also shown for the mean (dashed lines) \pm standard deviation (dotted lines). The white dots show the results of numerical simulations of the full model, without the oligomorphic approximation. Figures (b) and (c) show the equilibrium trait distributions and dynamics of mean traits in habitat $A$ (solid line) and $B$ (dashed line) obtained by numerical integration of a 2-morph oligomorphic approximation for $B A=0.4$ (b) or $m_{B A}=1.4$ (c). The dotted lines indicate the value of the class reproductive value of habitat $B$ for morphs 1 (blue) and 2 (orange), computed from the oligomorphic approximation. Parameter values: $b=1, g=2, m_{A B}=0.8, V_{M}=10^{-6}$. 
(a)
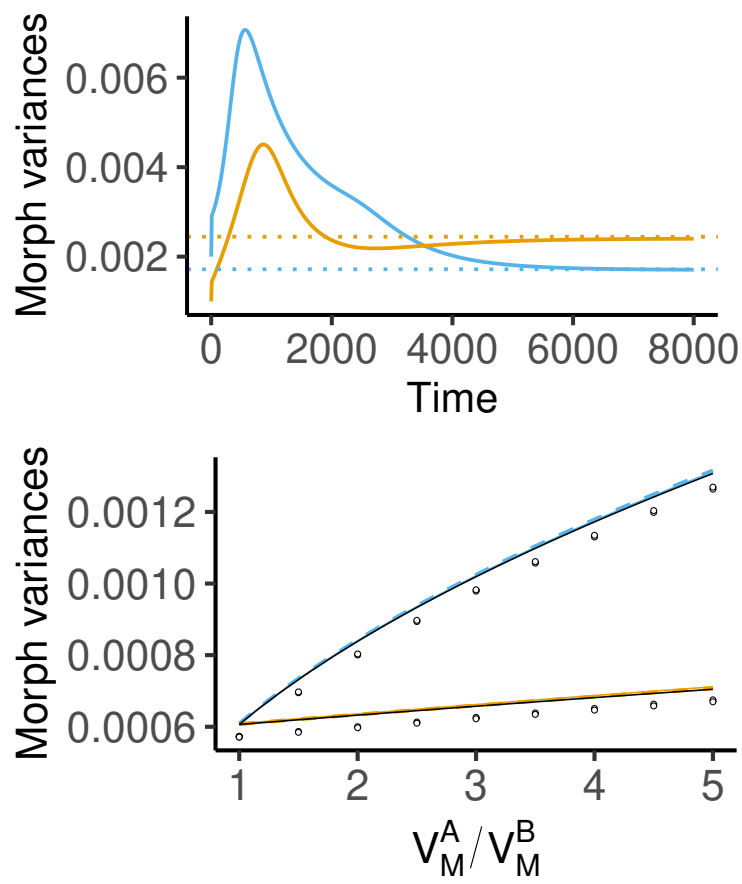

(b)

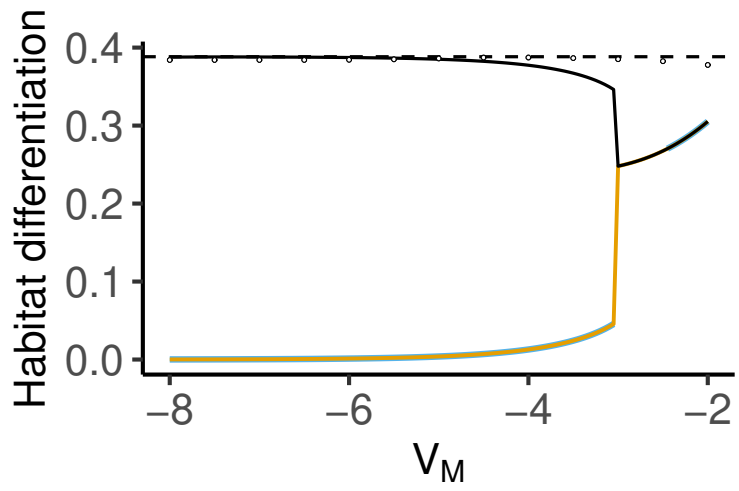

Figure 5: Effect of mutation. (a) Effect of habitat-specific mutation. The top panel shows the dynamics of the variances of morph 1 (blue) and 2 (orange) which converge to mutation-selection equilibrium values, which are accurately predicted by the reproductive-value-weighted formula (33) (dotted lines) with $V_{M}=c_{i}^{A} V_{M}^{A}+c_{i}^{B} V_{M}^{B}, V_{M}^{A}=5 \cdot 10^{-6}, V_{M}^{B}=10^{-6}$. The bottom panel shows the effect of the ratio $V_{M}^{A} / V_{M}^{B}$ on the variances $V_{i}^{A}$ (solid line) and $V_{i}^{B}$ (dashed line) for morphs 1 (blue) and 2 (orange). The white dots give the results of simulations of the full model without the oligomorphic approximation. (b) Effect of the magnitude of mutational variance on the mean habitat differentiation $D=\bar{z}^{B}-\bar{z}^{A}$ at equilibrium (black line). The dashed line gives the analytical prediction of equation (31), the white dots the simulations results, the blue and orange lines give the morph-specific habitat differentiation $\bar{z}_{i}^{B}-\bar{z}_{i}^{A}$. Parameters in all panels: $b=1, g=2, m_{A B}=0.8, m_{B A}=0.4$. 
lower-dimensional approximation of the eco-evolutionary dynamics of multi-morph structured populations. The combination of these two approximations allows us to obtain compact analytical expressions for the dynamics of multimodal trait distributions in structured populations under density- and frequency-dependent selection. These analytical results are biologically insightful as they highlight how the quality and quantity of individuals in different classes affect the eco-evolutionary dynamics.

At a general level, our theoretical framework lies at the intersection of population genetics, quantitative genetics and adaptive dynamics. First, as in population genetics, it predicts how the frequencies of interacting morphs change over time, but explictly takes into account eco-evolutionary feedbacks. Second, we also describe the dynamics of the mean and variance of the trait distribution of each morph. This is reminiscent of moment methods typically used in quantitative genetics (Barton \& Turelli, 1987; Turelli \& Barton, 1990; Barton \& Turelli, 1991), but our approach effectively extends these tools to multimodal distributions, frequency-dependent selection, and a broad range of ecological scenarios. Third, while our result for the dynamics of the mean takes the form of Lande's univariate theorem (Lande, 1976, 1979, 1982; Barfield et al., 2011), we also track the dynamics of genetic variance and describe how initially unimodal distributions can split into different modes due to frequency-dependent selection. This effectively bridges the gap between quantitative genetics and adaptative dynamics to provide a more complete understanding of the evolution of quantitative traits.

Our approach addresses the criticism of the very restrictive assumptions on mutation that are made in the adaptive dynamics literature and broadens the scope of classic quantitative genetics theory to examine the dynamics of multimode distributions and a wider range of ecological feedbacks. Classical quantitative genetics methods are typically restricted to unimodal character distributions. However multimodal distributions are a very common outcome when frequency dependence causes disruptive selection, as often occurs when ecological feedbacks are taken into account in evolutionary models. The possibility to handle disruptive selection has been a landmark of adaptive dynamics, but it relies on a restrictive assumption of rare mutations and small standing variance in the populations. Furthermore, most applications of adaptive dynamics focus on initially monomorphic populations, but the dynamics after evolutionary branching become less tractable. Our oligomorphic analysis will give equivalent results to adaptive dynamics in monomorphic populations, but makes the analysis of multimode populations, after branching, much easier, and it also allows us to take into account the effect of mutations and of substantial genetic variance at the population level.

While these technical advances were already present in Sasaki \& Dieckmann (2011)'s original paper, our extension to class structure makes our analysis directly applicable to a broad range of biological scenarios where individuals differ because of non-genetic factors such as age, physiological status, or spatial location. A drawback of this increased realism is that it inflates the number of ecological and genetic variables we need to track. We therefore apply recent theory on reproductive values (Lion, 2018a,b; Priklopil \& Lehmann, 2020) to simplify the oligomorphic analysis and obtain a compact description of how the morph-level trait distributions change over time when there are demographic transitions between classes. The key idea is to define a weighted trait distribution that gives us a way of examining how selection acts on a particular morph across all the classes (Fisher, 1930; Taylor \& Frank, 1996; Frank, 1998; Rousset, 2004; Lehmann \& Rousset, 2014; Lion, 2018a). For the dynamics of the mean trait of a morph, the result takes the form of the structured extension of Lande's theorem (Barfield et al., 2011), but we also provide a description of the dynamics of the morph variance. Application of the method to the simplified two-class case gives insight into how stabilizing and disruptive selection are impacted by class frequencies and reproductive values and shows when directional selection will impact disruptive selection. As such we provide a very general tractable framework for a more complete eco-evolutionary analysis of class-structured models.

In addition to these theoretical developements, we apply our approach to the classic two-habitat migration-selection model in order to illustrate its use in a concrete biological problem. We examined how migration and selection interplay to generate or prevent polymorphism when a population is using two habitats that differ in quality. The model is based on Ronce \& Kirkpatrick (2001) and Débarre et al. (2013), but we also examine asymmetric migration rates and examine the effect of habitat-specific mutation on evolutionary outcomes. We define a clear relationship between migration and selection that defines when disruptive selection is possible and show that our reproductive-value 
weighted oligomorphic approximation can accurately predict the numerical simulations of the full system. With the addition of mutation, the approach accurately predicts the equilibrium value of morph variances under-mutation selection balance. In addition we show that while mutation has no direct effect on morph means, since it impacts variances it can have an impact on the quantitative evolutionary outcome. This is because it impacts the rate of evolution and therefore in bistable regions mutation rates may determine whether disruptive selection occurs. Overall, the application of our approach to this specific model illustrates the accuracy of our approximations and allows us to build on previous analyses.

We expect our approach will allow us to deepen our understanding of the processes that generate and maintain diversity in traits. We illustrate the application of the approach in a two-habitat model, but this can be broadened to examine fundamental processes such as stage structure, species interactions, and speciation. For instance, the approach can be used to determine the time required until a population diversifies under frequency-dependent disruptive selection, which, for asexually reproducing species, is the waiting time until adaptive speciation. Additional insights will come from both ecological extensions - such as assortative mating - and in particular genetic extension - such as multi-locus inheritance, recombination, diploidy, and random genetic drift. We might expect the impact of diploidy to be even more important in multi-modal populations.

At a conceptual level, our approach is very relevant to the current revival of interest on the time scales of ecological and evolutionary processes, as it can be used to examine the role of 'fast evolution' when this is fueled by a large standing variation at the population level. In addition there is the potential to examine non-equilibrium outcomes such as those that arise during repeated epidemics driven by antigenic escape (Sasaki et al., 2021) or seasonality (Lion \& Gandon, 2021).

To sum up, we think our analytical approach will allow for a better understanding of the role of ecological feedbacks, frequency- and density-dependent selection in nature, and has the potential to facilitate a tighter integration between eco-evolutionary theory and empirical data. At a technical level, our approach moves the field on from either focusing on unimodal character distributions, often taken in models of quantitative genetics theory, or on negligible within-morph variance, which is often assumed in models of adaptive dynamics. At a biological level, it has considerable potential to advance our understanding of the ecological factors driving the evolution and maintenance of diversity, which remains an important empirical and theoretical challenge in multiple fields and contexts.

\section{Acknowledgements}

This study was supported by ANR JCJC grant ANR-16-CE35-0012-01 to SL, grants R01 GM12206103 and NSF-DEB-2011109 to MB, and the ESB Cooperation Program, The Graduate University for Advanced Studies, SOKENDAI.

\section{Appendix A: Oligomorphic approximation}

From equation (5) in the main text, it is straightforward to derive the dynamics of the within-class densities $\phi^{k}(z, t)=n^{k}(z, t) / n^{k}(t)$. We obtain

$$
\frac{\mathrm{d} \phi^{k}(z)}{\mathrm{d} t}=\sum_{j}\left[r^{k j}(z) \phi^{j}(z, t)-\bar{r}^{k j} \phi^{k}(z, t)\right] \frac{f^{j}}{f^{k}}
$$

To derive the oligomorphic approximatin for our class-structured population model, we proceed as in equation (6) of Sasaki \& Dieckmann, 2011 and define the dynamics of the frequencies $f_{i}^{k}$ so that, for each $z$, the contribution of morphs to $\mathrm{d} \phi(z) / \mathrm{d} t$ is proportional to their contribution to $\phi(z)$. We have

$$
\frac{\mathrm{d} f_{i}^{k} \phi_{i}^{k}(z)}{\mathrm{d} t}=\frac{f_{i}^{k} \phi_{i}^{k}(z)}{\phi^{k}(z)} \frac{\mathrm{d} \phi^{k}(z)}{\mathrm{d} t}
$$

Integrating over $z$ and using equation (A.1) yields

$$
\frac{\mathrm{d} f_{i}^{k}}{\mathrm{~d} t}=f_{i}^{k} \sum_{j} \frac{f^{j}}{f^{k}} \int r^{k j}(z) \frac{\phi^{j}(z)}{\phi^{k}(z)} \phi_{i}^{k}(z) \mathrm{d} z-f_{i}^{k} \sum_{j} \bar{r}^{k j} \frac{f^{j}}{f^{k}}
$$


To simplify this equation, we use the following important assumption:

$$
\frac{\phi^{j}(z)}{\phi^{k}(z)}=\frac{f_{i}^{j} \phi_{i}^{j}(z)}{f_{i}^{k} \phi_{i}^{k}(z)}
$$

which leads to

$$
\frac{\mathrm{d} f_{i}^{k}}{\mathrm{~d} t}=\sum_{j} \frac{f^{j}}{f^{k}}\left(f_{i}^{j} \bar{r}_{i}^{k j}-f_{i}^{k} \bar{r}^{k j}\right)
$$

With only one class, we recover equation (7) in Sasaki \& Dieckmann (2011).

Similarly, to calculate the dynamics of $\phi_{i}^{k}(z)$, we write

$$
\frac{\mathrm{d} \phi_{i}^{k}(z)}{\mathrm{d} t}=\frac{\phi_{i}^{k}(z)}{\phi^{k}(z)} \frac{\mathrm{d} \phi^{k}(z)}{\mathrm{d} t}-\phi_{i}^{k}(z)\left(\frac{1}{f_{i}^{k}} \frac{\mathrm{d} f_{i}^{k}}{\mathrm{~d} t}\right)
$$

Using equations (A.1), (A.3) and (A.4) then yields after some rearrangements:

$$
\frac{\mathrm{d} \phi_{i}^{k}(z)}{\mathrm{d} t}=\sum_{j} \frac{f_{i}^{j} f^{j}}{f_{i}^{k} f^{k}}\left[\phi_{i}^{j}(z) r^{k j}(z)-\phi_{i}^{k}(z) \bar{r}_{i}^{k j}\right]
$$

With a single class, we recover equation (8) in Sasaki \& Dieckmann (2011).

\section{Appendix B: Dynamics of class-specific morph moments}

Equation (A.5) can be multiplied by $z$ or $\left(z-\bar{z}_{i}^{k}\right)^{2}$ and integrated to obtain the dynamics of the morph means and variances respectively. For the morph means, we obtain:

$$
\frac{\mathrm{d} \bar{z}_{i}^{k}}{\mathrm{~d} t}=\sum_{j} \frac{f_{i}^{j} f^{j}}{f_{i}^{k} f^{k}} \operatorname{Cov}\left(z, r_{i}^{k j}(z)\right)+\sum_{j} \frac{f_{i}^{j} f^{j}}{f_{i}^{k} f^{k}} \bar{r}_{i}^{k j}\left(\bar{z}_{i}^{j}-\bar{z}_{i}^{k}\right)
$$

where the covariances are taken over the distributions $\phi_{i}^{j}(z, t)$ and take the form

$$
\underset{\phi_{i}^{j}}{\operatorname{Cov}}\left(z, r^{k j}(z)\right)=\int\left(z-\bar{z}_{i}^{j}\right)\left(r^{k j}(z)-\bar{r}_{i}^{k j}\right) \phi_{i}^{j}(z) \mathrm{d} z .
$$

Equation (B.1) is a morph-specific version of equation (3) in Lion (2018a). Using the Taylor expansion (6) in the main text, we have

$$
\begin{aligned}
\underset{\phi_{i}^{j}}{\operatorname{Cov}\left(z, r^{k j}(z)\right)} & =\int \xi_{i}^{j}\left(\left.\xi_{i}^{j} \frac{\partial r^{k j}}{\partial z}\right|_{z=\bar{z}_{i}^{j}}+\left.\frac{1}{2}\left(\left(\xi_{i}^{j}\right)^{2}-V_{i}^{j}\right) \frac{\partial^{2} r^{k j}}{\partial z^{2}}\right|_{z=\bar{z}_{i}^{j}}\right) \phi_{i}^{j}(z) \mathrm{d} z \\
& =\left.V_{i}^{j} \frac{\partial r^{k j}}{\partial z}\right|_{z=\bar{z}_{i}^{j}}+\left.\frac{1}{2} T_{i}^{j} \frac{\partial^{2} r^{k j}}{\partial z^{2}}\right|_{z=\bar{z}_{i}^{j}}+O\left(\varepsilon^{4}\right)
\end{aligned}
$$

where $T_{i}^{j}$ is the third central moment of $\phi_{i}^{j}(z)$, which we neglect in the following (assuming $\phi_{i}^{j}$ is symmetric). We then have

$$
\frac{\mathrm{d} \bar{z}_{i}^{k}}{\mathrm{~d} t}=\sum_{j} \frac{f_{i}^{j} f^{j}}{f_{i}^{k} f^{k}}\left[\left(\bar{z}_{i}^{j}-\bar{z}_{i}^{k}\right) r^{k j}\left(\bar{z}_{i}^{j}\right)+\left.V_{i}^{j} \frac{\partial r^{k j}}{\partial z}\right|_{z=\bar{z}_{i}^{j}}+\left.\frac{1}{2}\left(\bar{z}_{i}^{j}-\bar{z}_{i}^{k}\right) V_{i}^{j} \frac{\partial^{2} r^{k j}}{\partial z^{2}}\right|_{z=\bar{z}_{i}^{j}}\right]+O\left(\varepsilon^{4}\right)
$$

Keeping only terms up to second order in $\varepsilon$, we obtain equation (13) in the main text.

For the morph variances, defined as $V_{i}^{k}=\int\left(\xi_{i}^{k}\right)^{2} \phi_{i}^{k}(z) \mathrm{d} z$, we have, using equations (A.5) and (6)

$$
\begin{aligned}
\frac{\mathrm{d} V_{i}^{k}}{\mathrm{~d} t}=\sum_{j} & \frac{f_{i}^{j} f^{j}}{f_{i}^{k} f^{k}}\left[\left(\xi_{i}^{k}\right)^{2}\left(r^{k j}\left(\bar{z}_{i}^{j}\right)+\left.\xi_{i}^{j} \frac{\partial r^{k j}}{\partial z}\right|_{z=\bar{z}_{i}^{j}}+\left.\frac{1}{2}\left(\xi_{i}^{j}\right)^{2} \frac{\partial^{2} r^{k j}}{\partial z^{2}}\right|_{z=\bar{z}_{i}^{j}}\right) \phi_{i}^{j}(z) \mathrm{d} z\right. \\
& \left.-V_{i}^{k}\left(r^{k j}\left(\bar{z}_{i}^{j}\right)+\left.\frac{1}{2} V_{i}^{j} \frac{\partial^{2} r^{k j}}{\partial z^{2}}\right|_{z=\bar{z}_{i}^{j}}\right)\right]+O\left(\varepsilon^{5}\right) .
\end{aligned}
$$


We can further simplify with the following relationships:

$$
\begin{aligned}
\int\left(\xi_{i}^{k}\right)^{2} \phi_{i}^{j}(z) \mathrm{d} z & =\int\left(\xi_{i}^{j}+\bar{z}_{i}^{j}-\bar{z}_{i}^{k}\right)^{2} \phi_{i}^{j}(z) \mathrm{d} z=V_{i}^{j}+\left(\bar{z}_{i}^{j}-\bar{z}_{i}^{k}\right)^{2} \\
\int\left(\xi_{i}^{k}\right)^{2} \xi_{i}^{j} \phi_{i}^{j}(z) \mathrm{d} z & =\int\left(\xi_{i}^{3}+2\left(\bar{z}_{i}^{j}-\bar{z}_{i}^{k}\right)\left(\xi_{i}^{j}\right)^{2}+\left(\bar{z}_{i}^{j}-\bar{z}_{i}^{k}\right)^{2} \xi_{i}^{j}\right) \phi_{i}^{j}(z) \mathrm{d} z=T_{i}^{j}+2\left(\bar{z}_{i}^{j}-\bar{z}_{i}^{k}\right) V_{i}^{j} \\
\int\left(\xi_{i}^{k}\right)^{2}\left(\xi_{i}^{j}\right)^{2} \phi_{i}^{j}(z) \mathrm{d} z & =\int\left(\xi_{i}^{4}+2\left(\bar{z}_{i}^{j}-\bar{z}_{i}^{k}\right)\left(\xi_{i}^{j}\right)^{3}+\left(\bar{z}_{i}^{j}-\bar{z}_{i}^{k}\right)^{2}\left(\xi_{i}^{j}\right)^{2}\right) \phi_{i}^{j}(z) \mathrm{d} z=Q_{i}^{j}+2\left(\bar{z}_{i}^{j}-\bar{z}_{i}^{k}\right) T_{i}^{j}+\left(\bar{z}_{i}^{j}-\bar{z}_{i}^{k}\right)^{2} V_{i}^{j}
\end{aligned}
$$

This yields equation (18) in the main text, again using the assumption that the morph distribution $\phi_{i}^{j}$ is symmetric so that $T_{i}^{j}=0$.

\section{Appendix C: Dynamics of population-level morph moments}

The derivations in appendix B yield dynamical equations for the class-specific morph moments. Depending on the question of interest it may be useful to focus on the population-level morph moments, averaged over all classes. To do so, we introduce the following average distribution:

$$
\phi_{i}(z)=\sum_{k} \phi_{i}^{k}(z) u_{i}^{k}
$$

where $u_{i}^{k}=f_{i}^{k} f^{k} / f_{i}$ and $f_{i}=\sum_{k} f_{i}^{k} f^{k}$ is the total frequency of morph $i$ in the population. Integrating over $z$ leads to a relationship between morph means: $\bar{z}_{i}=\sum_{k} \bar{z}_{i}^{k} u_{i}^{k}$, from which the following equation can be derived

$$
\frac{\mathrm{d} \bar{z}_{i}}{\mathrm{~d} t}=\sum_{j} u_{i}^{j} \underset{\phi_{i}^{j}}{\operatorname{Cov}}\left(z, \sum_{k} r^{k j}(z)\right)+\sum_{j} u_{i}^{j}\left(\sum_{k} \bar{r}_{i}^{k j}\right)\left(\bar{z}_{i}^{j}-\bar{z}_{i}\right)
$$

This is the morph-specific equivalent of equation (2) in Lion (2018a), and an oligomorphic approximation of equation (C.1) can be derived using approximation (6). A similar equation can be derived for the dynamics of the morph variance, $V_{i}=\int\left(z-\bar{z}_{i}\right)^{2} \phi_{i}(z) \mathrm{d} z$.

\section{Appendix D: Projection on RV space}

In equation (C.1), the dynamics of $\bar{z}_{i}$ depends on the moments of the class-specific distributions $\phi_{i}^{j}(z)$. Our goal here is to find a meaningul way to summarise the dynamics using only the moments of the population-level morph distribution $\phi_{i}(z)$.

There are two equivalent ways to do this. The first approach applies the method of Lion (2018b) at the morph level, and uses a quasi-equilibrium approximation of the phenotypic differentiations $\bar{z}_{i}^{k}-\bar{z}_{i}$. This is summarised in Appendix S.1. The second, simpler approach is to calculate the moments of the reproductive-value-weighted distribution, as in Lion (2018a), but applied at the morph level. The time-dependent reproductive values satisfy equation (12), and the RV-weighted morph distribution is $\widetilde{\phi}_{i}(z)=\sum_{k} c_{i}^{k} \phi_{i}^{k}(z)$ where $c_{i}^{k}=v_{i}^{k} u_{i}^{k}$. Following Lion (2018a), we obtain the following Price equation:

$$
\frac{\mathrm{d} \widetilde{z}_{i}}{\mathrm{~d} t}=\sum_{j} \sum_{k} v_{i}^{k} \operatorname{Cov}_{\phi_{i}^{j}}\left(z, r^{k j}(z)\right) u_{i}^{j}
$$

So far, equation (D.1) is valid irrespective of the shape of the morph distribution. However, if the morph distribution is sufficiently narrow, we can approximate covariance using equation (B.2).

Both the QE and RV approaches lead to the following equation

$$
\frac{\mathrm{d} \widetilde{z}_{i}}{\mathrm{~d} t}=\left.\sum_{j} V_{i}^{j} \sum_{k} v_{i}^{k} \frac{\partial r^{k j}}{\partial z}\right|_{z=\bar{z}_{i}^{j}} u_{i}^{j}+O\left(\varepsilon^{3}\right) .
$$

Hence, for narrow morph distributions, the morph mean and the RV-weighted morph mean have the same dynamics on the slow manifold characterised by $\overline{\mathbf{R}} \mathbf{n}=\overline{\mathbf{R}} \mathbf{f}=\overline{\mathbf{R}}_{i} \mathbf{u}_{i}=\mathbf{v}_{i}^{\top} \overline{\mathbf{R}}_{i}=\mathbf{0}$. 
Importantly, the RHS of equation (D.2) still depends on the class-specific moments $\bar{z}_{i}^{j}$ and $V_{i}^{j}$. However, after relaxation on the RV space, the quantities $\bar{z}_{i}^{k}-\widetilde{z}_{i}$ and $V_{i}^{k}-\widetilde{V}_{i}$ will typically be $O\left(\varepsilon^{2}\right)$ and $O\left(\varepsilon^{4}\right)$ respectively under the oligomorphic approximation. Hence, to leading order, we can replace the habitat-specific morph means and variances by the corresponding moments of the RV-weighted distribution. This substitution thus introduces a small error, but it will be quantitatively acceptable as long as the morph variances remain small. This will notably be the case near evolutionary endpoints under stabilising selection. With this last approximation, we obtain equation (25) in the main text.

We can also calculate the dynamics of the morph variances, either using a quasi-equilibrium approach (as shown in Appendix S.2 for a two-class model), or by calculating the dynamics of the $\mathrm{RV}$-weighted morph variances $\widetilde{V}_{i}$. This latter approach yields the folowing Price equation

$$
\frac{\mathrm{d} \widetilde{V}_{i}}{\mathrm{~d} t}=\sum_{j} \sum_{k} v_{i}^{k} \operatorname{Cov}_{\phi_{i}^{j}}\left(\left(z-\widetilde{z}_{i}\right)^{2}, r^{k j}(z)\right) u_{i}^{j} .
$$

For narrow morph distributions, this can be approximated as

$$
\left.\frac{\mathrm{d} \widetilde{V}_{i}}{\mathrm{~d} t} \approx \sum_{j} \sum_{k} v_{i}^{k} \frac{Q_{i}^{j}-\left(V_{i}^{j}\right)^{2}}{2} \frac{\partial^{2} r^{k j}}{\partial z^{2}}\right|_{z=\bar{z}_{i}^{j}} u_{i}^{j}+\left.2 \sum_{j} V_{i}^{j} \sum_{k} v_{i}^{k} \frac{\partial r^{k j}}{\partial z}\right|_{z=\bar{z}_{i}^{j}}\left(\bar{z}_{i}^{j}-\widetilde{z}_{i}\right) u_{i}^{j}+O\left(\varepsilon^{5}\right)
$$

With the Gaussian closure approximation $Q_{i}^{j}=3\left(V_{i}^{j}\right)^{2}$ and again using $\bar{z}_{i}^{k}-\widetilde{z}_{i}=O\left(\varepsilon^{2}\right)$ and $V_{i}^{k}-\widetilde{V}_{i}=$ $O\left(\varepsilon^{4}\right)$, we obtain

$$
\frac{\mathrm{d} \widetilde{V}_{i}}{\mathrm{~d} t} \approx 2 V_{i}^{2}\left[\left.\frac{1}{2} \sum_{j} \sum_{k} v_{i}^{k} \frac{\partial^{2} r^{k j}}{\partial z^{2}}\right|_{z=\widetilde{z}_{i}} u_{i}^{j}+\left.\sum_{j} \sum_{k} v_{i}^{k} \frac{\partial r^{k j}}{\partial z}\right|_{z=\widetilde{z}_{i}}\left(\bar{z}_{i}^{j}-\widetilde{z}_{i}\right) u_{i}^{j}\right]+O\left(\varepsilon^{5}\right)
$$

which can be rewritten in matrix form as equation (20) in the main text.

\section{References}

Abrams, P. A., H. Matsuda \& Y. Harada (1993). Evolutionarily unstable fitness maxima and stable fitness minima of continuous traits. Evol. Ecol. 7(5): 465-487.

Abrams, P. A. (2001). Modelling the adaptive dynamics of traits involved in inter- and intraspecific interactions: An assessment of three methods. Ecol. Lett. 4: 166-175. DOI: $10.1046 / \mathrm{j} .1461-$ 0248.2001.00199.x.

Barfield, M., R. D. Holt \& R. Gomulkiewicz (2011). Evolution in stage-structured populations. Am. Nat. 177(4): 397-409. DOI: 10.1086/658903.

Barton, N. H. \& M. Turelli (1991). Natural and sexual selection on many loci. Genetics. 127(1): 229255.

Barton, N. H. \& M. Turelli (1987). Adaptive landscapes, genetic distance and the evolution of quantitative characters. Genetical Research. 49: 157-173. DOI: 10.1017/S0016672300026951.

Bonamour, S., C. Teplitsky, A. Charmantier, P. A. Crochet \& L. M. Chevin (2017). Selection on skewed characters and the paradox of stasis. Evolution. 71(11): 2703-2713. DOI: 10.1111/evo.13368.

Bulmer, M. G. (1992). The mathematical theory of quantitative genetics. Oxford University Press, Oxford.

Crow, J. F. \& M. Kimura (1970). An Introduction to Population Genetics Theory. Burgess Publishing Company, Minneapolis, MN, USA.

Day, T. (2005). Modeling the ecological context of evolutionary change: déjà vu or something new? In: Ecological paradigms lost: Routes to theory change. Ed. by Cuddington, K. \& Beisnet, B. E. Academic Press.

Débarre, F., O. Ronce \& S. Gandon (2013). Quantifying the effects of migration and mutation on adaptation and demography in spatially heterogeneous environments. J. evol. Biol. 26(6): 11851202. ISSN: $1420-9101$. DOI: $10.1111 / \mathrm{jeb} .12132$.

Dieckmann, U. \& R. Law (1996). The dynamical theory of coevolution: a derivation from stochastic ecological processes. Journal of mathematical biology. 34(5): 579-612. DOI: 10.1007/BF02409751. 
Ewens, W. J. (2004). Mathematical Population Genetics. Springer-Verlag, New York.

Falconer, D. S. (1996). Introduction to quantitative genetics. Longman, Harlow.

Fisher, R. A. (1930). The genetical theory of natural selection. Clarendon Press, Oxford, UK.

Frank, S. A. (1998). Foundations of social evolution. Princeton University Press, Princeton, NJ, USA.

Gardner, A. (2015). The genetical theory of multilevel selection. J. evol. Biol. 28(2): 305-319. ISSN: 1420-9101. DOI: $10.1111 / \mathrm{jeb} .12566$.

Geritz, S. A. H., É. Kisdi, G. Meszéna \& J. A. J. Metz (1998). Evolutionarily singular strategies and the adaptive growth and branching of the evolutionary tree. Evol. Ecol. 12: 35-57. DOI: 10. 1023/A: 1006554906681.

Grafen, A. (2015). Biological fitness and the Price equation in class-structured populations. J. theor. Biol. 373: 62-72. DOI: 10.1016/j.jtbi.2015.02.014.

Iwasa, Y., A. Pomiankowski \& S. Nee (1991). The evolution of costly mate preferences: II the "handicap" principle. Evolution. 45: 1431-1442.

Kimura, M. (1965). A stochastic model concerning the maintenance of genetic variability in quantitative characters. P. Natl. Acad. Sci. USA. 54(3): 732-736. DOI: 10.1073/pnas .54.3.731.

Lande, R. (1975). The maintenance of genetic variability by mutation in a polygenic character with linked loci. Genetical Research. 26(3): 221-235.

Lande, R. (1976). Natural selection and random genetic drift in phenotypic evolution. Evolution. 30: $314-334$.

Lande, R. (1979). Quantitative genetic analysis of multivariate evolution, applied to brain-body size allometry. Evolution. 33: 402-416.

Lande, R. (1982). A quantitative genetic theory of life history evolution. Ecology. 63(3): 607-615.

Lehmann, L. \& F. Rousset (2014). The genetical theory of social behaviour. Phil. Trans. Roy. Soc. B. 369(1642). DOI: 10.1098/rstb.2013.0357.

Lion, S. (2018a). Class structure, demography and selection: reproductive-value weighting in nonequilibrium, polymorphic populations. Am. Nat. 191(5): 620-637. DOI: https://doi .org/10 . $1101 / 155879$.

Lion, S. (2018b). From the Price equation to the selection gradient in class-structured populations: a quasi-equilibrium route. J. theor. Biol. 447C: 178-189. DOI: 10.1016/j.jtbi.2018.03.033.

Lion, S. (2018c). Theoretical approaches in evolutionary ecology: environmental feedback as a unifying perspective. Am. Nat. 191(1): 21-44. DOI: 10.1086/694865.

Lion, S. \& S. Gandon (2021). Life-history evolution of class-structured populations in fluctuating environments. bioRxiv: 2021.03.12.435065. DOI: 10.1101/2021.03.12.435065.

Meszéna, G., I. Czibula \& S. Geritz (1997). Adaptive Dynamics in a 2-Patch Environment: A Toy Model for Allopatric and Parapatric Speciation. Journal of Biological Systems. 5(2): 265-284. DOI: 10.1142/S0218339097000175.

Metz, J. A. J., S. A. H. Geritz, G. Meszéna, F. J. A. Jacobs \& J. S. van Heerwaarden (1996). Adaptive dynamics: A geometrical study of the consequences of nearly faithful reproduction. In: Stochastic and spatial structures of dynamical systems. Ed. by van Strien, S. J. \& Verduyn Lunel, S. M. North-Holland, Amsterdam, The Netherlands, pp. 183-231.

Metz, J. A. J., S. D. Mylius \& O. Diekmann (2008). When does evolution optimize? Evol. Ecol. Res. 10(5): 629-654.

Metz, J. A. J., R. M. Nisbet \& S. A. H. Geritz (1992). How should we define 'fitness' for general ecological scenarios? Trends Ecol. Evol. 7(6): 198-202. DOI: 10.1016/0169-5347(92)90073-K.

Mirrahimi, S. \& S. Gandon (2020). Evolution of specialization in heterogeneous environments: equilibrium between selection, mutation and migration. DOI: 10.1534/genetics.119.302868.

Mylius, S. D. \& O. Diekmann (1995). On evolutionarily stable life histories, optimization and the need to be specific about density dependence. Oikos. 74: 218-224. DOI: 10.2307/3545651.

Priklopil, T. \& L. Lehmann (2020). Invasion implies substitution in ecological communities with classstructured populations. Theor. Pop. Biol. 134: 36-52. DOI: 10.1016/j.tpb.2020.04.004.

Ronce, O. \& M. Kirkpatrick (2001). When sources become sinks: migrational meltdown in heterogeneous habitats. Evolution. 55(8): 1520-1531.

Rousset, F. (1999). Reproductive value vs sources and sinks. Oikos. 86(3): 591-596. 
Rousset, F. (2004). Genetic structure and selection in subdivided populations. Princeton University Press, Princeton, NJ, USA.

Sasaki, A. \& U. Dieckmann (2011). Oligomorphic dynamics for analyzing the quantitative genetics of adaptive speciation. J. math. Biol. 63(4): 601-35. DOI: 10.1007/s00285-010-0380-6.

Sasaki, A., S. Lion \& M. Boots (2021). The impact of antigenic escape on the evolution of virulence. bioRxiv: 2021.01.19.427227. DOI: 10.1101/2021.01.19.427227.

Taylor, P. D. (1990). Allele-frequency change in a class-structured population. Am. Nat. 135(1): 95106. DOI: $10.1086 / 285034$.

Taylor, P. D. \& S. A. Frank (1996). How to make a kin selection model? J. theor. Biol. 180(1): 27-37. DOI: $10.1006 / j$ tbi.1996.0075.

Turelli, M. \& N. H. Barton (1990). Dynamics of polygenic characters under selection. Theor. Pop. Biol. 38(1): 1-57. DOI: 10.1016/0040-5809(90)90002-D.

Walsh, B. \& M. Lynch (2018). Evolution and selection of quantitative traits. Oxford University Press, Oxford. 


\section{Appendix S: Supplementary Material}

\section{S.1 Projection on RV space: quasi-equilibrium approach}

Let us define $\mathbf{u}_{i}$ the vector with elements $f_{i}^{k} f^{k} / f_{i}, \mathbf{U}_{i}=\operatorname{diag}\left(\mathbf{u}_{i}\right), \mathbf{C}_{i}$ the matrix with elements $\operatorname{Cov}\left(z, r^{k j}(z)\right)$, and $\mathbf{d}_{i}$ the vector of morph-specific scaled phenotypic differentiation (with elements $d_{i}^{k}=\left(\bar{z}_{i}^{k}-\bar{z}_{i}\right) / \sigma_{i}$ where $\sigma_{i}=\sqrt{V_{i}}$ is the standard deviation of the distribution of morph $\left.i\right)$. We can then write equation (C.1) as

$$
\frac{\mathrm{d} \bar{z}_{i}}{\mathrm{~d} t}=\mathbf{1}^{\top} \mathbf{C}_{i} \mathbf{u}_{i}+\sigma_{i} \mathbf{1}^{\top} \overline{\mathbf{R}}_{i} \mathbf{U}_{i} \mathbf{d}_{i}
$$

which has the same form as equation (15) in Lion (2018b), but is morph-specific. Similarly, the dynamics of $\mathbf{d}_{i}$ can be put in the form:

$$
\frac{\mathrm{d}\left(\sigma_{i} \mathbf{d}_{i}\right)}{\mathrm{d} t}=\mathbf{B}_{i} \mathbf{C}_{i} \mathbf{u}_{i}+\mathbf{A}_{i}\left(\sigma_{i} \mathbf{d}_{i}\right)
$$

which has the same form as equation (A2) in Lion (2018b). Hence, if we assume, as in Lion (2018b), that the unimodal morph distributions are tightly clustered around the mean (which is the crux of the oligomorphic approximation), we can follow the same approach as in that paper, and derive a quasi-equilibrium approximation for $\mathbf{d}_{i}$. This eventually yields:

$$
\frac{\mathrm{d} \bar{z}_{i}}{\mathrm{~d} t} \approx \mathbf{v}_{i}^{\top} \mathbf{S}_{\mathbf{i}} \boldsymbol{\Omega}_{i} \mathbf{u}_{i}+O\left(\varepsilon^{3}\right)
$$

where $\mathbf{v}_{i}^{\top}$ and $\mathbf{u}_{i}$ are calculated as the left and right eigenvectors of $\mathbf{R}_{i}$, keeping only the $O(1)$ terms, $\boldsymbol{\Omega}_{i}=\operatorname{diag}\left(V_{i}^{1} \ldots V_{i}^{K}\right)$ and the matrix $\mathbf{S}_{i}$ has elements $\partial r^{k j} / \partial z$ evaluated at $z=\bar{z}_{i}^{j}$. To leading order, we can replace $\bar{z}_{i}^{j}$ and $V_{i}^{j}$ by $\bar{z}_{i}$ and $V_{i}$ (because both $\bar{z}_{i}^{j}-\bar{z}_{i}$ and $V_{i}^{j}-V_{i}$ are at least $O(\varepsilon)$ ) to obtain equation (17) in the main text.

\section{S.2 A two-habitat model}

In this appendix, we carry out an explicit analysis of a specific two-habitat model to revisit the results of Débarre et al. (2013). As in that paper, we consider a population of individuals distributed over two habitats, $A$ and $B$, coupled by migration. Each habitat is characterised by a habitat-specific optimum $\left(\theta_{A}\right.$ and $\theta_{B}$, respectively). The transition rates between classes are then

$$
\begin{aligned}
& r^{A A}(z)=b-n^{A}-g\left(z-\theta_{A}\right)^{2}-m_{B A} \\
& r^{A B}(z)=m_{A B} \\
& r^{B A}(z)=m_{B A} \\
& r^{B B}(z)=b-n^{B}-g\left(z-\theta_{B}\right)^{2}-m_{A B}
\end{aligned}
$$

where $b$ is the fecundity rate and $g$ is the fecundity cost. We use quadratic cost functions for simplicity, so that the cost is minimal at the habitat's optimum. Also, in contrast to Débarre et al. (2013), we consider asymmetric migration rates, with $m_{j k}$ the migration rate from habitat $k$ to habitat $j$ (see also Mirrahimi \& Gandon (2020)). Note that we assume migration rates do not depend on the focal trait, which will lead to simplifications as the partial derivatives of $r^{k j}(z)$ will vanish for $j \neq k$. 


\section{S.2.1 Oligomorphic approximation}

Using the general equations derived in the main text, we obtain the following equations for the oligomorphic dynamics

$$
\begin{aligned}
& \frac{\mathrm{d}}{\mathrm{d} t}\left(\begin{array}{c}
n^{A} \\
n^{B}
\end{array}\right)=\left(\begin{array}{cc}
\sum_{i} f_{i}^{A} r^{A A}\left(\bar{z}_{i}^{A}\right) & m_{A B} \\
m_{B A} & \sum_{i} f_{i}^{B} r^{B B}\left(\bar{z}_{i}^{B}\right)
\end{array}\right)\left(\begin{array}{c}
n^{A} \\
n^{B}
\end{array}\right)+O\left(\varepsilon^{2}\right) \\
& \frac{\mathrm{d} f_{i}^{A}}{\mathrm{~d} t}=f_{i}^{A}\left[r^{A A}\left(\bar{z}_{i}^{A}\right)-\sum_{\ell} f_{\ell}^{A} r^{A A}\left(\bar{z}_{\ell}^{A}\right)\right]+\frac{n^{B}}{n^{A}} m_{A B}\left[f_{i}^{B}-f_{i}^{A}\right]+O\left(\varepsilon^{2}\right) \\
& \frac{\mathrm{d} f_{i}^{B}}{\mathrm{~d} t}=f_{i}^{B}\left[r^{B B}\left(\bar{z}_{i}^{B}\right)-\sum_{\ell} f_{\ell}^{B} r^{B B}\left(\bar{z}_{\ell}^{B}\right)\right]+\frac{n^{A}}{n^{B}} m_{B A}\left[f_{i}^{A}-f_{i}^{B}\right]+O\left(\varepsilon^{2}\right) \\
& \frac{\mathrm{d} \bar{z}_{i}^{A}}{\mathrm{~d} t}=\left.V_{i}^{A} \frac{\partial r^{A A}}{\partial z}\right|_{z=\bar{z}_{i}^{A}}+\frac{f_{i}^{B} n^{B}}{f_{i}^{A} n^{A}} m_{A B}\left(\bar{z}_{i}^{B}-\bar{z}_{i}^{A}\right)+O\left(\varepsilon^{3}\right) \\
& \frac{\mathrm{d} \bar{z}_{i}^{B}}{\mathrm{~d} t}=\left.V_{i}^{B} \frac{\partial r^{B B}}{\partial z}\right|_{z=\bar{z}_{i}^{B}}+\frac{f_{i}^{A} n^{A}}{f_{i}^{B} n^{B}} m_{B A}\left(\bar{z}_{i}^{A}-\bar{z}_{i}^{B}\right)+O\left(\varepsilon^{3}\right) \\
& \frac{\mathrm{d} V_{i}^{A}}{\mathrm{~d} t}=\left.\frac{1}{2}\left[Q_{i}^{A}-\left(V_{i}^{A}\right)^{2}\right] \frac{\partial^{2} r^{A A}}{\partial z^{2}}\right|_{z=\bar{z}_{i}^{A}}+\frac{f_{i}^{B} n^{B}}{f_{i}^{A} n^{A}} m_{A B}\left[V_{i}^{B}-V_{i}^{A}+\left(\bar{z}_{i}^{B}-\bar{z}_{i}^{A}\right)^{2}\right]+O\left(\varepsilon^{5}\right) \\
& \frac{\mathrm{d} V_{i}^{B}}{\mathrm{~d} t}=\left.\frac{1}{2}\left[Q_{i}^{B}-\left(V_{i}^{B}\right)^{2}\right] \frac{\partial^{2} r^{B B}}{\partial z^{2}}\right|_{z=\bar{z}_{i}^{B}}+\frac{f_{i}^{A} n^{A}}{f_{i}^{B} n^{B}} m_{B A}\left[V_{i}^{A}-V_{i}^{B}+\left(\bar{z}_{i}^{A}-\bar{z}_{i}^{B}\right)^{2}\right]+O\left(\varepsilon^{5}\right)
\end{aligned}
$$

together with the approximations

$$
\begin{aligned}
r^{k k}\left(\bar{z}_{i}^{k}\right) & =b-w_{k}\left(\bar{z}_{i}^{k}\right)-m_{k}-n^{k} \\
\left.\frac{\partial r^{k k}}{\partial z}\right|_{z=\bar{z}_{i}^{k}} & =-w_{k}^{\prime}\left(\bar{z}_{i}^{k}\right) \\
\left.\frac{\partial^{2} r^{k k}}{\partial z^{2}}\right|_{z=\bar{z}_{i}^{k}} & =-w_{k}^{\prime \prime}\left(\bar{z}_{i}^{k}\right)
\end{aligned}
$$

where $w_{A}(z)=g\left(z-\theta_{A}\right)^{2}, w_{B}(z)=g\left(z-\theta_{B}\right)^{2}, m_{A}=m_{B A}$ and $m_{B}=m_{A B}$

Reproductive values This system can be numerically solved, but we can get some further simplifications using reproductive values. For a two-class model, class reproductive values satisfy at quasi-equilibrium

$$
c_{i}^{B}=\frac{m_{A B}\left(f_{i}^{B} f^{B}\right)^{2}}{m_{A B}\left(f_{i}^{B} f^{B}\right)^{2}+m_{B A}\left(f_{i}^{A} f^{A}\right)^{2}}=1-c_{i}^{A}
$$

where the morph and class frequencies are calculated using the $O(1)$ terms of equations (S.4a)-(S.4c).

Morph means Using equations (S.3) and (D.1), it is straightforward to derive the following equation for the dynamics of the morph mean, $\bar{z}_{i}$, and the RV-weighted morph mean $\widetilde{z}_{i}$. We obtain

$$
\frac{\mathrm{d} \bar{z}_{i}}{\mathrm{~d} t}=\frac{\mathrm{d} \widetilde{z}_{i}}{\mathrm{~d} t}=\left.c_{i}^{A} V_{i}^{A} \frac{\partial r^{A A}}{\partial z}\right|_{z=\bar{z}_{i}^{A}}+\left.c_{i}^{B} V_{i}^{B} \frac{\partial r^{B B}}{\partial z}\right|_{z=\bar{z}_{i}^{B}}+O\left(\varepsilon^{3}\right)
$$

If we only want to keep $O\left(\varepsilon^{2}\right)$ terms, it is sufficient to replace $V_{i}^{A}$ and $V_{i}^{B}$ by $V_{i}$, the morph variance, and $\bar{z}_{i}^{A}$ and $\bar{z}_{i}^{B}$ by the morph mean $\bar{z}_{i}$. (See below for an explicit justification.) We then obtain for our model:

$$
\frac{\mathrm{d} \bar{z}_{i}}{\mathrm{~d} t}=-V_{i}\left[c_{i}^{A} u_{A}^{\prime}\left(\bar{z}_{i}\right)+c_{i}^{B} u_{B}^{\prime}\left(\bar{z}_{i}\right)\right]+O\left(\varepsilon^{3}\right) \approx-2 g V_{i}\left[\bar{z}_{i}-c_{i}^{A} \theta_{A}-c_{i}^{B} \theta_{B}\right]
$$




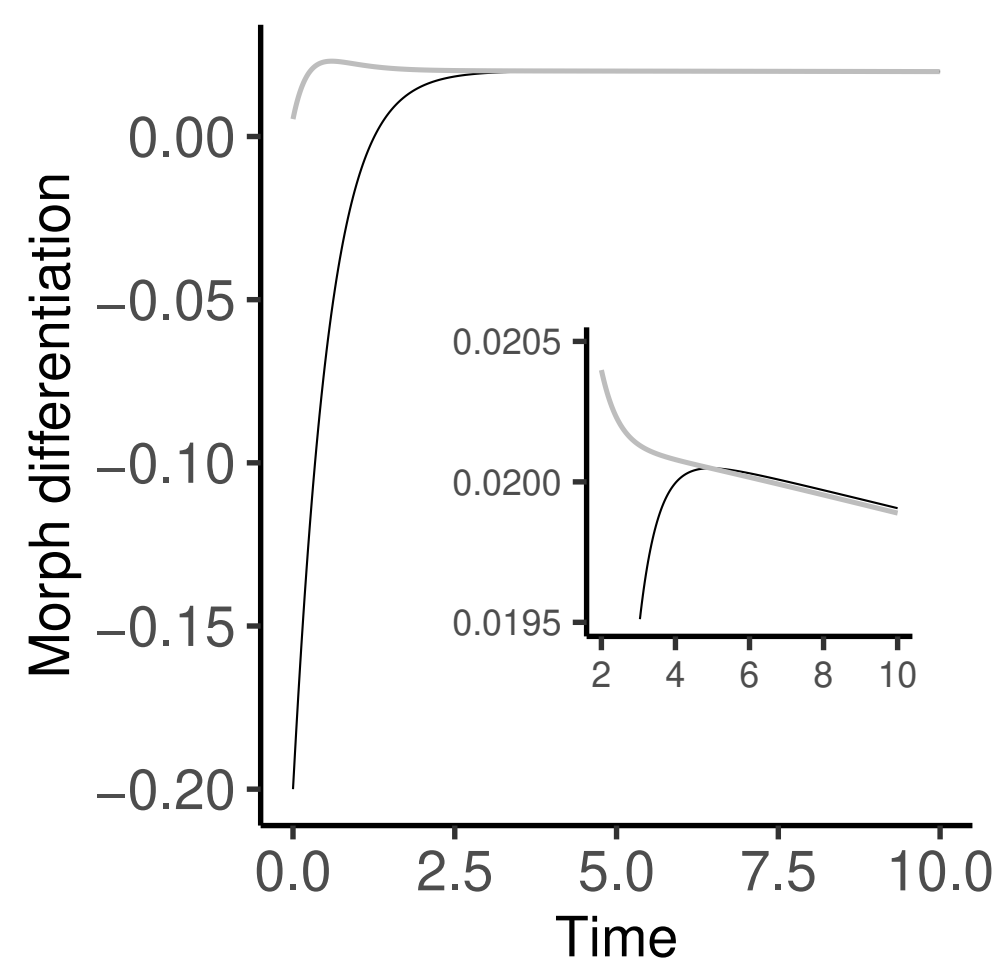

Figure S.1: Illustration of the relaxation to RV space in a two-class model. The simulation is the same as in figure S.3a, to which the reader is referred for additional details. The morph differentiation $\bar{z}_{B, 1}-$ $\bar{z}_{A, 1}$ (black) is shown to converge towards the value predicted by the quasi-equilibrium approximation (S.9) (grey line). The inset shows a close-up of the dynamics.

An explicit derivation of equation (S.7) can also be obtained by calculating the dynamics of $\bar{z}_{i}^{B}-\bar{z}_{i}^{A}$ from equations (S.4d)-(S.4e) and assuming this phenotypic differentiation is a fast variable (Lion, 2018b). This yields the following quasi-equilibrium approximation

$$
\bar{z}_{i}^{B}-\bar{z}_{i}^{A} \approx \frac{f_{i}^{A} f^{A} f_{i}^{B} f^{B}}{m_{A B}\left(f_{i}^{B} f^{B}\right)^{2}+m_{B A}\left(f_{i}^{A} f^{A}\right)^{2}}\left(\left.V_{i}^{B} \frac{\partial r^{B B}}{\partial z}\right|_{z=\bar{z}_{i}^{B}}-\left.V_{i}^{A} \frac{\partial r^{A A}}{\partial z}\right|_{z=\bar{z}_{i}^{A}}\right)
$$

which can be written using equation (S.6) as

$$
\bar{z}_{i}^{B}-\bar{z}_{i}^{A} \approx \sqrt{\frac{c_{i}^{A} c_{i}^{B}}{m_{A B} m_{B A}}}\left(\left.V_{i}^{B} \frac{\partial r^{B B}}{\partial z}\right|_{z=\bar{z}_{i}^{B}}-\left.V_{i}^{A} \frac{\partial r^{A A}}{\partial z}\right|_{z=\bar{z}_{i}^{A}}\right)
$$

Plugging the resulting expression into equations (S.4d) and (S.4e) yields

$$
\frac{\mathrm{d} \bar{z}_{i}^{A}}{\mathrm{~d} t}=\frac{\mathrm{d} \bar{z}_{i}^{B}}{\mathrm{~d} t}=\left.c_{i}^{A} V_{i}^{A} \frac{\partial r^{A A}}{\partial z}\right|_{z=\bar{z}_{i}^{A}}+\left.c_{i}^{B} V_{i}^{B} \frac{\partial r^{B B}}{\partial z}\right|_{z=\bar{z}_{i}^{B}}+O\left(\varepsilon^{3}\right)
$$

which entails, assuming that the morph and class frequencies are calculated on their quasi-equilibrium manifold,

$$
\frac{\mathrm{d} \bar{z}_{i}}{\mathrm{~d} t}=\frac{\mathrm{d} \widetilde{z}_{i}}{\mathrm{~d} t}=\frac{\mathrm{d} \bar{z}_{i}^{A}}{\mathrm{~d} t}=\frac{\mathrm{d} \bar{z}_{i}^{B}}{\mathrm{~d} t}
$$

Equation (S.10) shows that $\bar{z}_{i}^{B}-\bar{z}_{i}^{A}=O\left(\varepsilon^{2}\right)$, so that $\bar{z}_{i}^{A}=\bar{z}_{i}^{B}=\bar{z}_{i}+O\left(\varepsilon^{2}\right)$. This justifies that we can replace the class-specific morph means by the morph mean in equation (S.7). We then obtain equation (25) in the main text. Figure S.1 shows that the differentiation between morph means $\bar{z}_{B, 1}-\bar{z}_{A, 1}$ quickly converges to a small value which is well predicted by the quasi-equilibrium approximation. 
Morph variances Similarly, we can derive the dynamics of the difference in morph variances from equations (S.4f)-(S.4g). With Gaussian closure approximation (such that $Q_{i}^{k}=3\left(V_{i}^{k}\right)^{2}$ ) and using equation (S.6), we obtain after some rearrangements the following quasi-equilibrium approximation:

$$
V_{i}^{B}-V_{i}^{A} \approx \sqrt{\frac{c_{i}^{A} c_{i}^{B}}{m_{A B} m_{B A}}}\left[\left.\left(V_{i}^{B}\right)^{2} \frac{\partial^{2} r^{B B}}{\partial z^{2}}\right|_{z=\bar{z}_{i}^{B}}-\left.\left(V_{i}^{A}\right)^{2} \frac{\partial^{2} r^{A A}}{\partial z^{2}}\right|_{z=\bar{z}_{i}^{A}}\right]-\left(c_{i}^{B}-c_{i}^{A}\right)\left(\bar{z}_{i}^{B}-\bar{z}_{i}^{A}\right)^{2}
$$

Plugging this into equations (S.4f) and (S.4g) yields, again after some rearrangements

$$
\frac{\mathrm{d} V_{i}^{A}}{\mathrm{~d} t}=\frac{\mathrm{d} V_{i}^{B}}{\mathrm{~d} t}=\left.c_{i}^{A}\left(V_{i}^{A}\right)^{2} \frac{\partial^{2} r^{A A}}{\partial z^{2}}\right|_{z=\bar{z}_{i}^{A}}+\left.c_{i} B\left(V_{i}^{B}\right)^{2} \frac{\partial^{2} r^{B B}}{\partial z^{2}}\right|_{z=\bar{z}_{i}^{B}}+2 \sqrt{c_{i}^{A} c_{i}^{B} m_{A B} m_{B A}}\left(\bar{z}_{i}^{B}-\bar{z}_{i}^{A}\right)^{2}
$$

and using equation (S.10) finally yields

$$
\begin{aligned}
\frac{\mathrm{d} V_{i}^{A}}{\mathrm{~d} t}=\frac{\mathrm{d} V_{i}^{B}}{\mathrm{~d} t}= & \left.c_{i}^{A}\left(V_{i}^{A}\right)^{2} \frac{\partial^{2} r^{A A}}{\partial z^{2}}\right|_{z=\bar{z}_{i}^{A}}+\left.c_{i}^{B}\left(V_{i}^{B}\right)^{2} \frac{\partial^{2} r^{B B}}{\partial z^{2}}\right|_{z=\bar{z}_{i}^{B}} \\
& +2 \frac{\left(c_{i}^{A} c_{i}^{B}\right)^{3 / 2}}{\sqrt{m_{A B} m_{B A}}}\left(\left.V_{i}^{B} \frac{\partial r^{B B}}{\partial z}\right|_{z=\bar{z}_{i}^{B}}-\left.V_{i}^{A} \frac{\partial r^{A A}}{\partial z}\right|_{z=\bar{z}_{i}^{A}}\right)^{2}
\end{aligned}
$$

which again implies

$$
\frac{\mathrm{d} V_{i}}{\mathrm{~d} t}=\frac{\mathrm{d} \widetilde{V}_{i}}{\mathrm{~d} t}=\frac{\mathrm{d} V_{i}^{A}}{\mathrm{~d} t}=\frac{\mathrm{d} V_{i}^{B}}{\mathrm{~d} t}
$$

Another route to this result is to start with the dynamics of the RV-weighted variance, given by equation (20). This gives for our model

$$
\left.\frac{\mathrm{d} \widetilde{V}_{i}}{\mathrm{~d} t} \approx c_{i}^{A}\left(V_{i}^{A}\right)^{2} \frac{\partial^{2} r^{A A}}{\partial z^{2}}\right|_{z=\bar{z}_{i}^{A}}+\left.c_{i}^{B}\left(V_{i}^{B}\right)^{2} \frac{\partial^{2} r^{B B}}{\partial z^{2}}\right|_{z=\bar{z}_{i}^{B}}+\left.2 c_{i}^{A}\left(\bar{z}_{i}^{A}-\widetilde{z}_{i}\right) V_{i}^{A} \frac{\partial r^{A A}}{\partial z}\right|_{z=\bar{z}_{i}^{A}}+\left.2 c_{i}^{B}\left(\bar{z}_{i}^{B}-\widetilde{z}_{i}\right) V_{i}^{B} \frac{\partial r^{A A}}{\partial z}\right|_{z=\bar{z}_{i}^{B}}
$$

(recall that $\left.v_{i}^{k} u_{i}^{k}=c_{i}^{k}\right)$. Noting that $\bar{z}_{i}^{A}-\widetilde{z}_{i}=c_{i}^{B}\left(\bar{z}_{i}^{A}-\bar{z}_{i}^{B}\right)$ and $\bar{z}_{i}^{B}-\widetilde{z}_{i}=c_{i}^{A}\left(\bar{z}_{i}^{B}-\bar{z}_{i}^{A}\right)$, and using equation (S.10) finally yields

$$
\begin{aligned}
\frac{\mathrm{d} \widetilde{V}_{i}}{\mathrm{~d} t}= & \left.c_{i}^{A}\left(V_{i}^{A}\right)^{2} \frac{\partial^{2} r^{A A}}{\partial z^{2}}\right|_{z=\bar{z}_{i}^{A}}+\left.c_{i}^{B}\left(V_{i}^{B}\right)^{2} \frac{\partial^{2} r^{B B}}{\partial z^{2}}\right|_{z=\bar{z}_{i}^{B}} \\
& +2 \frac{\left(c_{i}^{A} c_{i}^{B}\right)^{3 / 2}}{\sqrt{m_{A B} m_{B A}}}\left(\left.V_{i}^{B} \frac{\partial r^{B B}}{\partial z}\right|_{z=\bar{z}_{i}^{B}}-\left.V_{i}^{A} \frac{\partial r^{A A}}{\partial z}\right|_{z=\bar{z}_{i}^{A}}\right)^{2}
\end{aligned}
$$

From equation (S.12) we see that $V_{i}^{B}-V_{i}^{A}=O\left(\tilde{\widetilde{V}}^{4}\right)$, so that $V_{i}^{A}=V_{i}^{B}=\widetilde{V}_{i}+O\left(\varepsilon^{4}\right)$. The errors made by approximating the class-specific variances by $\widetilde{V}_{i}$ in equation (S.16) will therefore be of higher-order than the leading-order terms.

\section{S.2.2 Quadratic functions}

For the model with quadratic functions, equations (S.7) and (S.16) simplify to

$$
\begin{aligned}
\frac{\mathrm{d} \bar{z}_{i}}{\mathrm{~d} t} & =-2 g V_{i}\left[\bar{z}_{i}-c_{i}^{A} \theta_{A}-c_{i}^{B} \theta_{B}\right] \\
\frac{\mathrm{d} V_{i}}{\mathrm{~d} t} & =-2 g V_{i}^{2}\left[1-\frac{4 g}{\sqrt{m_{A B} m_{B A}}}\left(c_{i}^{A} c_{i}^{B}\right)^{3 / 2}\left(\theta_{B}-\theta_{A}\right)^{2}\right]
\end{aligned}
$$

Equation (S.17) shows that at equilibrium morph means are equal to the reproductive-value weighted average of habitat optima, $c_{i}^{A} \theta_{A}+c_{i}^{B} \theta_{B}$. The corresponding equilibra are stable if $\mathrm{d} V_{i} / \mathrm{d} t<0$, which is equivalent to

$$
\frac{\sqrt{m_{A B} m_{B A}}}{4 g}>\left(c_{i}^{A} c_{i}^{B}\right)^{3 / 2}
$$

where we have set $\theta_{A}=0=1-\theta_{B}$ without loss of generality. 


\section{S.2.3 Solutions}

We consider that the population is composed of two morphs (1 and 2). We can thus write the dynamics of the frequencies of morph 1 in habitats $A$ and $B$ as follows:

$$
\begin{aligned}
& 0=\left(f_{1}^{A}\left(1-f_{1}^{A}\right) g\left(\bar{z}_{1}-\bar{z}_{2}\right)\left(\bar{z}_{1}+\bar{z}_{2}\right)+m_{A B} \frac{f^{B}}{f^{A}}\left(f_{1}^{B}-f_{1}^{A}\right)\right. \\
& 0=\left(f_{1}^{B}\left(1-f_{1}^{B}\right) g\left(\bar{z}_{1}-\bar{z}_{2}\right)\left(\bar{z}_{1}+\bar{z}_{2}-2\right)+m_{B A} \frac{f^{A}}{f^{B}}\left(f_{1}^{A}-f_{1}^{B}\right)\right.
\end{aligned}
$$

Multiplying the first equation by $f^{A} /\left(m_{A B} f^{B}\right)$ and the second by $f^{B} /\left(m_{B A} f^{A}\right)$, then taking the sum, gives

$$
0=g\left(\bar{z}_{1}-\bar{z}_{2}\right)\left[\frac{f^{A} f_{1}^{A}\left(1-f_{1}^{A}\right)}{m_{A B} f^{B}}\left(\bar{z}_{1}+\bar{z}_{2}\right)+\frac{f^{B} f_{1}^{B}\left(1-f_{1}^{B}\right)}{m_{B A} f^{A}}\left(\bar{z}_{1}+\bar{z}_{2}-2\right)\right]
$$

This is satisfied either if $\bar{z}_{1}=\bar{z}_{2}$ which corresponds to a single-morph equilibrium, or if the term between brackets is zero, which leads to a dimorphic equilibrium

Dimorphic equilibrium We start with the dimorphic case, which is simpler to analyse. The term between brackets yields

$$
\frac{f_{1}^{B}\left(1-f_{2}^{B}\right)}{f_{1}^{A}\left(1-f_{1}^{A}\right)}=\frac{\bar{z}_{1}+\bar{z}_{2}}{2-\bar{z}_{1}-\bar{z}_{2}} \frac{m_{B A}}{m_{A B}}\left(\frac{f^{A}}{f^{B}}\right)^{2}
$$

Using the fact that $\bar{z}_{i} /\left(1-\bar{z}_{i}\right)=c_{i}^{B} / c_{i}^{A}=\left(m_{A B} / m_{B A}\right)\left(f_{i}^{B} f^{B} /\left(f_{i}^{A} f^{A}\right)\right)^{2}$, we can simplify this equality as

$$
\frac{\bar{z}_{1} \bar{z}_{2}}{\left(1-\bar{z}_{1}\right)\left(1-\bar{z}_{2}\right)}=\left(\frac{\bar{z}_{1}+\bar{z}_{2}}{2-\bar{z}_{1}-\bar{z}_{2}}\right)^{2}
$$

which can be rearranged as

$$
\left(\bar{z}_{1}-\bar{z}_{2}\right)^{2}\left(1-\bar{z}_{1}-\bar{z}_{2}\right)=0
$$

Hence, because $\bar{z}_{1} \neq \bar{z}_{2}$ for the dimorphic equilibrium, the morph means must satisfy $\bar{z}_{1}+\bar{z}_{2}=1$. Plugging this condition into the dynamics of morph frequencies, then solving for $f_{1}^{A}$ and $f_{1}^{B}$ and calculating $c_{1}^{B}$ yields

$$
\bar{z}_{1}=c_{1}^{B}=\frac{1}{2}-\frac{g\left(1-2 \bar{z}_{1}\right)}{2 \sqrt{4 m_{A B} m_{B A}+g^{2}\left(1-2 \bar{z}_{1}^{2}\right)}}
$$

Solving for $\bar{z}_{1}$ finally yields, for $\sqrt{m_{A B} m_{B A}}<g / 2$

$$
\bar{z}_{1}=\frac{1}{2}-\frac{1}{2} \sqrt{1-\frac{4 m_{A B} m_{B A}}{g^{2}}}=1-\bar{z}_{2}
$$

which, using equation (S.18), is evolutionarily stable if $\sqrt{m_{A B} m_{B A}}<g / 2$.

Equation (S.22) can be used to calculate the morph frequencies (see companinon Mathematica notebook). We also obtain the following expressions for the equilibrium densities

$$
\begin{aligned}
& n^{A}=b-m_{B A}+\frac{m_{A B} m_{B A}}{g} \\
& n^{B}=b-m_{A B}+\frac{m_{A B} m_{B A}}{g}
\end{aligned}
$$

For symmetric migration, we recover the results in Table 1 of Débarre et al. (2013).

Because the dimorphic equilibrium is characterised by the two morphs having different frequencies in the two habitats, the habitat-specific trait distributions are distinct. We can characterise these equilibrium distributions by calculating their moments (see companion notebook): 
(a)

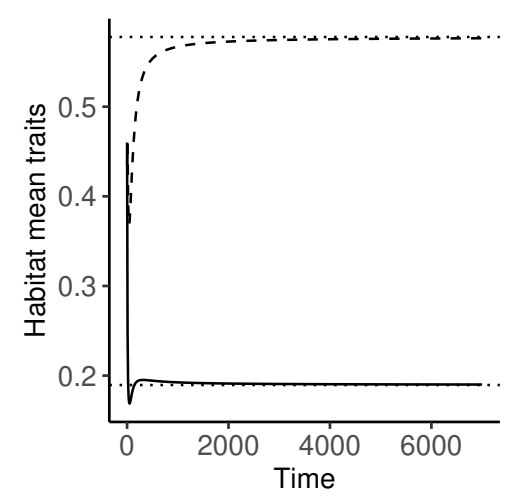

(b)

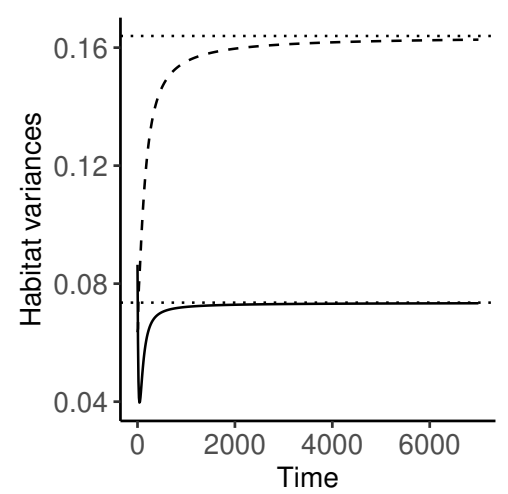

(c)

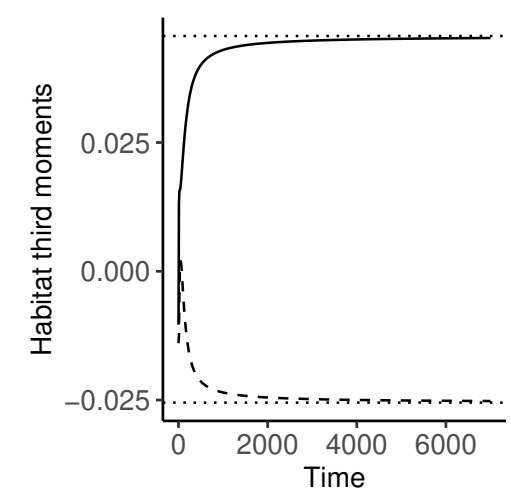

Figure S.2: Dynamics of habitat-specific moments: the means (a), variances (b) and third moments (c) of the trait distributions in habitat A (solid lines) and B (dashed lines) and shown to converge to the values predicted by the analytical formulae (dotted horizontal lines). Parameters as in figure $4 \mathrm{~b}$ in the main text.

- the mean trait in habitats $A$ and $B$ (and the differentiation $D=\bar{z}^{B}-\bar{z}^{A}$ )

$$
\begin{aligned}
& \bar{z}^{A}=f_{1}^{A} \bar{z}_{1}+\left(1-f_{1}^{A}\right) \bar{z}_{2}=\frac{m_{A B} f^{B}}{g f^{A}} \\
& \bar{z}^{B}=f_{1}^{B} \bar{z}_{1}+\left(1-f_{1}^{B}\right) \bar{z}_{2}=1-\frac{m_{B A} f^{A}}{g f^{B}}
\end{aligned}
$$

- the global mean trait

$$
\bar{z}=f^{A} \bar{z}^{A}+f^{B} \bar{z}^{B}=f^{B}+\frac{m_{A B} f^{B}-m_{B A} f^{A}}{g}
$$

where the first term is the mean of the two optima $\left(f^{A} \theta_{A}+f^{B} \theta_{B}=f^{B}\right)$ and the second term is the deviation caused by the migration-selection balance. For symmetric migration, we have $\bar{z}=f^{B}=1 / 2$.

- the variance in habitat $A$ in the absence of mutation-selection balance (i.e. assuming $V_{i}^{A}=0$ at equilibrium)

$$
V^{A}=\left(\bar{z}_{1}-\bar{z}^{A}\right)^{2} f_{1}^{A}+\left(\bar{z}_{2}-\bar{z}^{B}\right)^{2}\left(1-f_{1}^{A}\right)=m_{A B} \frac{f^{A} f^{B} g-m_{A B}\left(f^{B}\right)^{2}-m_{B A}\left(f^{A}\right)^{2}}{\left(f^{A} g\right)^{2}}
$$

- the third moment in habitat $A$ in the absence of mutation-selection balance (i.e. assuming $V_{i}^{A}=0$ at equilibrium), assuming the morph distribution is not skewed (e.g. $T_{i}^{A}=0$ )

$$
T^{A}=\left(\bar{z}_{1}-\bar{z}^{A}\right)^{3} f_{1}^{A}+\left(\bar{z}_{2}-\bar{z}^{B}\right)^{3}\left(1-f_{1}^{A}\right)=\frac{f^{A} g-2 f^{B} m_{A B}}{f^{A} g} V^{A}
$$

Note that, for symmetric migration, we recover the results of Débarre et al. (2013) (column 2 in their Table 1). In figure S.2, the dynamics of the moments of the trait distributions in habitats A and B are presented and compared with the analytical predictions.

Single-morph equilibria In the single-morph case, we have only one morph with frequencies $f_{1}^{A}=$ $f_{1}^{B}=1$. The equilibrium densities and morph mean can be calculated from the following system of 
equations:

$$
\begin{aligned}
\frac{\mathrm{d} n^{A}}{\mathrm{~d} t} & =\left(b-n^{A}-g \bar{z}_{1}^{2}-m_{B A}\right) n^{A}+m_{A B} n^{B}=0 \\
\frac{\mathrm{d} n^{B}}{\mathrm{~d} t} & =\left(b-n^{B}-g\left(\bar{z}_{1}-1\right)^{2}-m_{A B}\right) n^{B}+m_{B A} n^{A}=0 \\
\bar{z}_{1} & =\frac{m_{A B}\left(n^{B}\right)^{2}}{m_{A B}\left(n^{B}\right)^{2}+m_{B A}\left(n^{A}\right)^{2}}
\end{aligned}
$$

where the latter equation simply states that the mean trait is equal to the class reproductive value $c_{1}^{B}$.

The system can only be fully solved numerically, except for symmetric migration where at least one solution $\left(\bar{z}_{1}=1 / 2\right.$ and $\left.n^{A}=n^{B}=b-g / 4\right)$ can be analytically calculated. Depending on the region of parameter space (and in particular the values of the migration rates) there is typically either one or three solutions of the system.

Note that, in the limit where $m_{A B}=m_{B A}=m$, we have $v_{1}^{A}=v_{1}^{B}=1$ and therefore $\bar{z}_{1}=c_{1}^{B}=1 / 2$. The "symmetric monomorphic" singularity found by Débarre et al. (2013) thus corresponds to the case where both habitats have equal reproductive values. As found by Débarre et al. (2013), this solution is evolutionarily stable if $m>g / 2$, which can be checked using condition (S.18).

Bistability For some parameters values, the system can exhibit several convergent and evolutionarily stable points, notably the dimorphic equilibrium and one or two single-morph equilibria. The endpoint of the eco-evolutionary dynamics is then determined by the initial conditions. This bistability is illustrated in figure S.3 for a specific example, and the full bifurcation diagrams of the model for $m_{A B}=0.8$ are shown in figures S.4. Note however that, as already found by Débarre et al. (2013), the basin of attraction of the unimodal equilibrium is relatively narrow so that a little mutation is sufficient to push the dynamics towards the bimodal equilibrium. This explains why the simulations of the full model (the black dots in figures S.4a and S.4b) typically converge towards the bimodal equilibrium when it exists.

\section{(a) Dimorphic equilibrium}

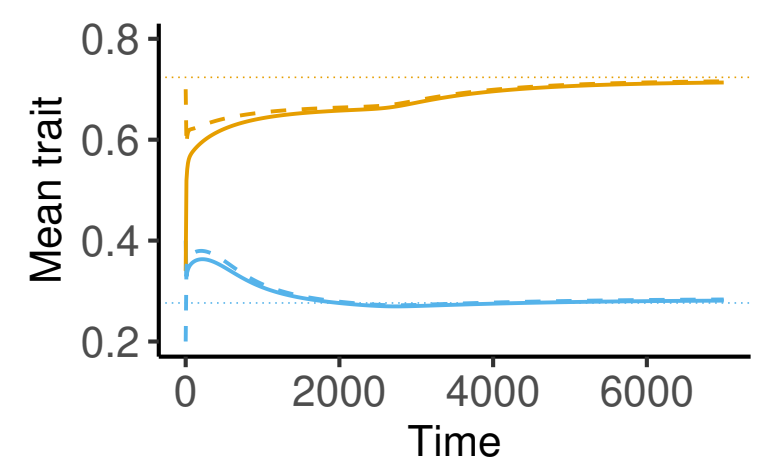

(b) Single-morph equilibrium

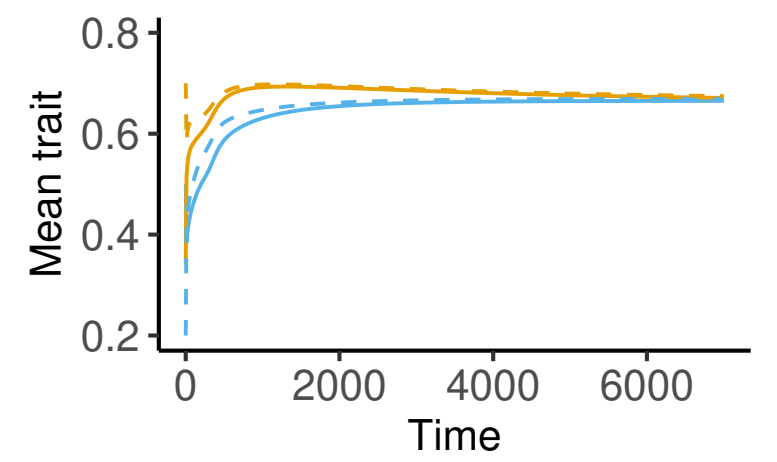

Figure S.3: Illustration of the bistable dynamics of the model for sufficiently low values of the geometric mean of the migration rate, $m=\sqrt{m_{A B} m_{B A}}$. The dynamics of the mean trait of morph 1 (blue) and 2 (black) in habitat A (solid lines) and B (dashed lines) are shown, either leading to a polymorphic equilibrium (left panel) or to a monomorphic equilibrium (panel b). The only difference between the two simulations is the initial trait value of the first morph in habitat $\mathrm{A}$, which is $x_{1}^{A}(0)=0.4$ in panel $(\mathrm{a})$, and $x_{1}^{A}(0)=0.5$ in panel (b). Other initial conditions: $n_{A}(0)=n_{B}(0)=1, f_{i}^{k}(0)=1 / 2$ (for $i=1,2$ and $k=A, B$ ), $x_{2}^{A}(0)=0.34, x_{1}^{B}(0)=0.2, x_{2}^{B}(0)=0.7, V_{1}^{A}(0)=V_{1}^{B}(0)=0.002$, $V_{2}^{A}(0)=V_{2}^{B}(0)=0.001$. Parameters: $m_{A B}=0.8, m_{B A}=1, g=2, b=1$.

\section{S.2.4 Box 1 vs. Box 2}

How accurate is it to replace the equations in Box 1 by the projection of RV space (Box 2)? Figure S.4 shows that the quantitative match is very good, except in a small region of parameter space between 
(a) Dimorphic equilibrium

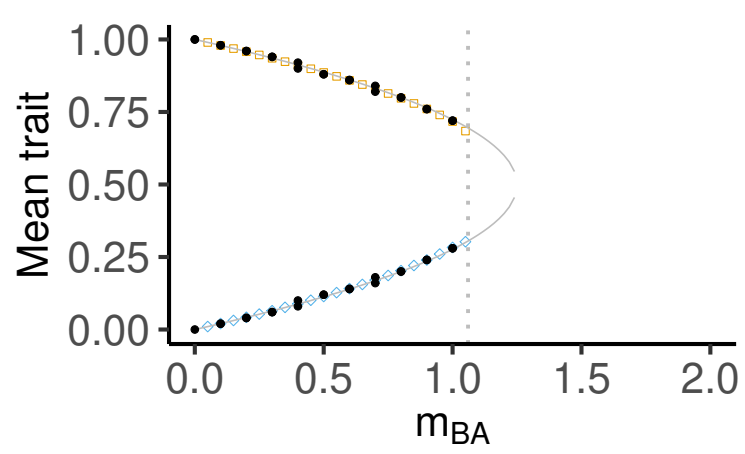

(b) Single-morph equilibria

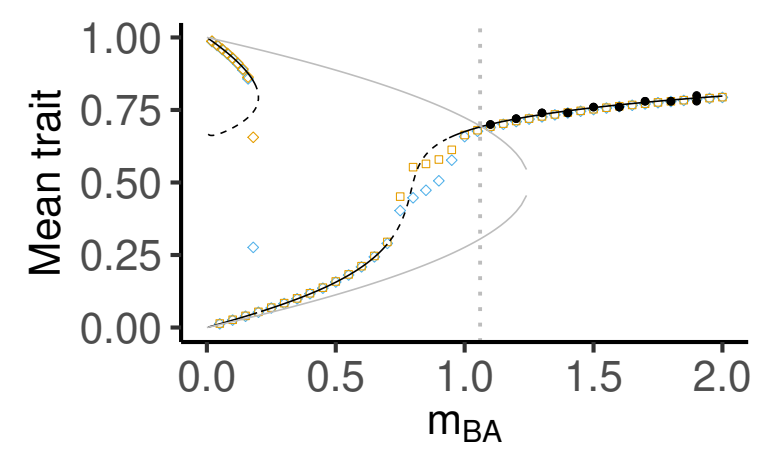

Figure S.4: Bistability. Figures (a) and (b) give the bifurcation diagrams for the mean traits. Open shapes give the predictions of a two-morph oligomorphic approximation for the dimorphic (circles, panel b) and single-morph (diamonds, panel c) solutions, for both morphs 1 (blue) and 2 (orange). The gray lines represent the analytical expressions $1 / 2 \pm \sqrt{1-4 m^{2} / g^{2}} / 2$, which are shown on both panels (b) and (c) for convenience. The results of the full model, without the oligomorphic approximation, are presented using black dots. On panel (b), the black lines give the predictions of the oligomorphic approximation (solid lines represent evolutionarily stable, and dashed lines evolutionarily unstable solutions). In all panels, the vertical dotted line represents the value $m_{B A} \approx 1.06$ at which the dimorphic equilibrium loses its demographic stability and one of the two morphs goes extinct. Parameter values: $b=1, g=2, m_{A B}=0.8, V_{M}=10^{-6}$.

$m_{A B}=0.8$ and $m_{B A}=1$, where the RV projection does not accurately predicts the single-morph solution. This corresponds to a point where migration is close to symmetric and the single-morph solution actually becomes a repellor. Thus the dynamics converge towards a point where selection is disruptive but a dimorphism cannot persist. As shown in figure S.5b, this causes the build-up of subtantial differentiation between the morph means in habitats $A$ and $B$, at which point the morphcentred reproductive-value-weighted oligomorphic approximation breaks down.

\section{S.2.5 Effect of mutation}

In figure S.6, we show how the mutational variance affects the equilibrium trait distributions in habitat A (top panel) and B (middle panel), and the morph frequencies (bottom panel). There is a sharp change in behaviour around $V_{M} \approx 10^{-3}$, which corresponds to the oligomorphic approximation breaking down when the morph variances become too large and collide: we then shift from a dimorphism with two distinct morphs (distinct frequencies of morph 1 and 2 in each habitat, but the morph means are the same in each habitat $\bar{z}_{i}^{A}=\bar{z}_{i}^{B}=\bar{z}_{i}$ ) to a case where one morph suddenly goes extinct, but this morph has a distinct mean in each habitat (i.e. $\bar{z}_{1}^{A} \neq \bar{z}_{1}^{B}$ ). However, the simulations of the full model do not predict this pattern, but rather than the model always converges towards a bimodal distributions with two peaks, albeit with slightly wider variances when $V_{M}$ is larger. Since the oligomorphic approximation relies on morph variances (i.e. the width of the peaks) being small enough, this is an expected behaviour of our approach. Nonetheless, the oligomorphic approximation remains accurate for relatively large mutational variance. 
(a)

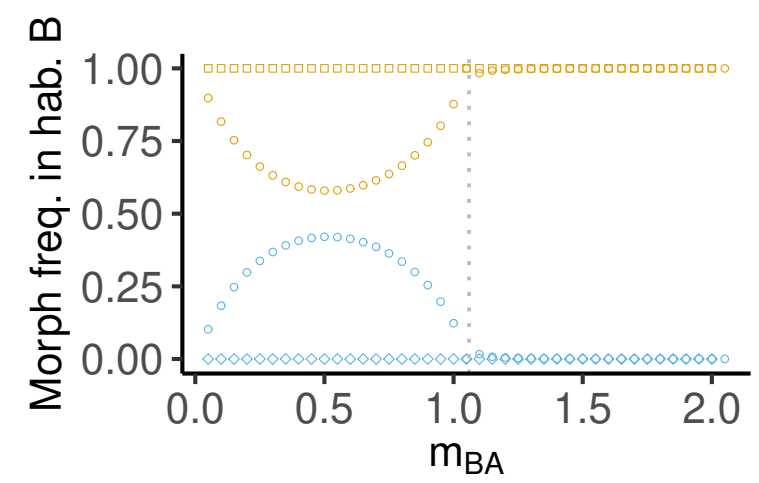

(b)

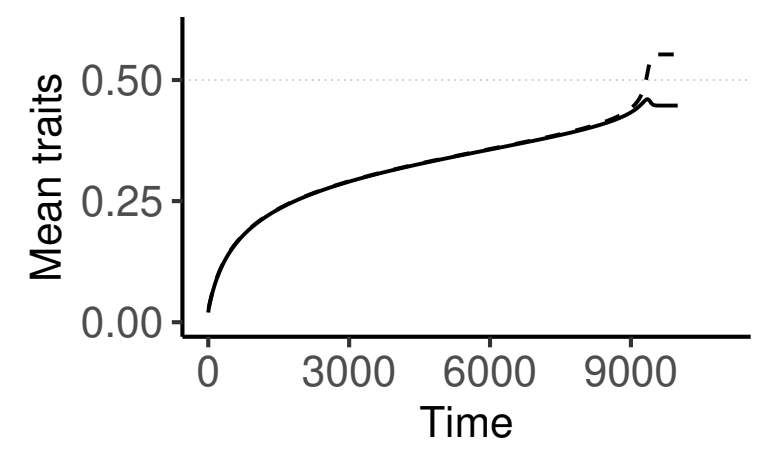

Figure S.5: This figure gives some additional results which are helpful to better understand figure S.4 Panel (a) gives the corresponding equilibrium values of the frequencies of morphs 1 (blue) and 2 (orange) for the single-morph (diamonds) and two-morph (circles) equilibria. Panel (b) represents the dynamics of the mean trait in habitat $A$ (solid line) and $B$ (dashed line) predicted by the single-morph oligomorphic approximation for $m_{A B}=m_{B A}=0.8$. In all panels, the vertical dotted line represents the value $m_{B A} \approx 1.06$ at which the dimorphic equilibrium loses its demographic stability and one of the two morphs goes extinct. Parameters as in figure 4.

\section{S.3 A two-habitat resource-competition model}

In this appendix, we extend the previous migration-habitat model to add trait-mediated resource competition. The transition rates between classes become

$$
\begin{aligned}
& r^{A A}(z)=b-n^{A} \int a(z-y) \phi^{A}(y, t) \mathrm{d} y-g\left(z-\theta_{A}\right)^{2}-m \\
& r^{A B}(z)=m \\
& r^{B A}(z)=m \\
& r^{B B}(z)=b-n^{B} \int a(z-y) \phi^{B}(y, t) \mathrm{d} y-g\left(z-\theta_{B}\right)^{2}-m
\end{aligned}
$$

where $a(\cdot)$ is the competition kernel. In the case where only one habitat is present, this corresponds to the model studied by Sasaki \& Dieckmann (2011).

\section{Deriving the dynamical equations}

In this model, the dynamics of morph means and variances are also described by equations (S.7) and (S.16), but we use the oligomorphic approximation to calculate the derivatives of the rates $r^{A A}(z)$ and $r^{B B}(z)$. As in Sasaki \& Dieckmann (2011), we first Taylor-expand the competition kernel around $y=\bar{z}_{\ell}^{j}$ to obtain

$$
a(z-y)=a\left(z-\bar{z}_{\ell}^{j}\right)+a^{\prime}\left(z-\bar{z}_{\ell}^{j}\right)\left(y-\bar{z}_{\ell}^{j}\right)+\frac{1}{2} a^{\prime \prime}\left(z-\bar{z}_{\ell}^{j}\right)\left(y-\bar{z}_{\ell}^{j}\right)^{2}+O\left(\varepsilon^{3}\right)
$$

Multiplying by $f_{\ell}^{j} \phi_{\ell}^{j}(y, t)$ and summing over $\ell$ yields

$$
a(z-y) \phi^{j}(y, t)=\sum_{\ell} f_{\ell}^{j} a\left(z-\bar{z}_{\ell}^{j}\right) \phi_{\ell}^{j}(y, t)+\sum_{\ell} f_{\ell}^{j} a^{\prime}\left(z-\bar{z}_{\ell}^{j}\right)\left(y-\bar{z}_{\ell}^{j}\right) \phi_{\ell}^{j}(y, t)+\frac{1}{2} \sum_{\ell} f_{\ell}^{j} a^{\prime \prime}\left(z-\bar{z}_{\ell}^{j}\right)\left(y-\bar{z}_{\ell}^{j}\right)^{2} \phi_{\ell}^{j}(y, t)+O\left(\varepsilon^{3}\right)
$$

Integrating over $y$, we obtain:

$$
\int a(z-y) \phi^{j}(y, t) \mathrm{d} y=\sum_{\ell} f_{\ell}^{j} a\left(z-\bar{z}_{\ell}^{j}\right)+\frac{1}{2} \sum_{\ell} f_{\ell}^{j} V_{\ell}^{j} a^{\prime \prime}\left(z-\bar{z}_{\ell}^{j}\right)+O\left(\varepsilon^{3}\right)
$$




\section{(a) Trait distribution in habitat $\mathbf{A}$}

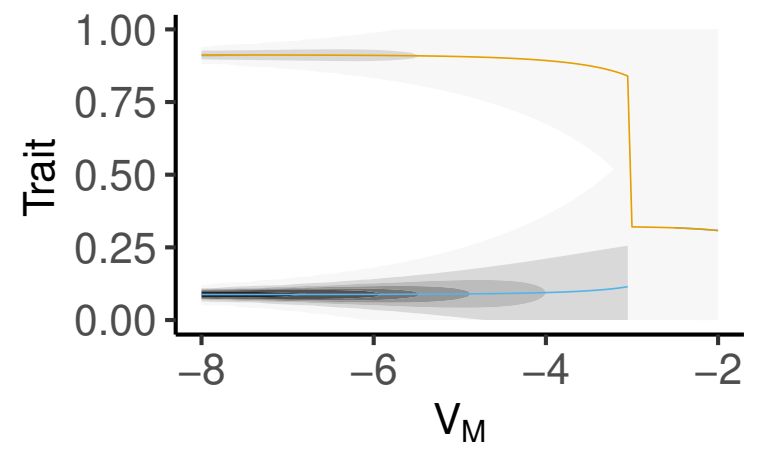

(b) Trait distribution in habitat $B$

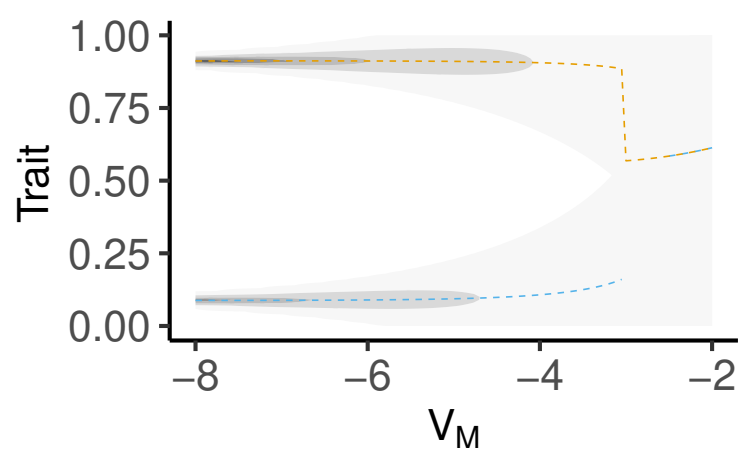

(c) Morph frequencies

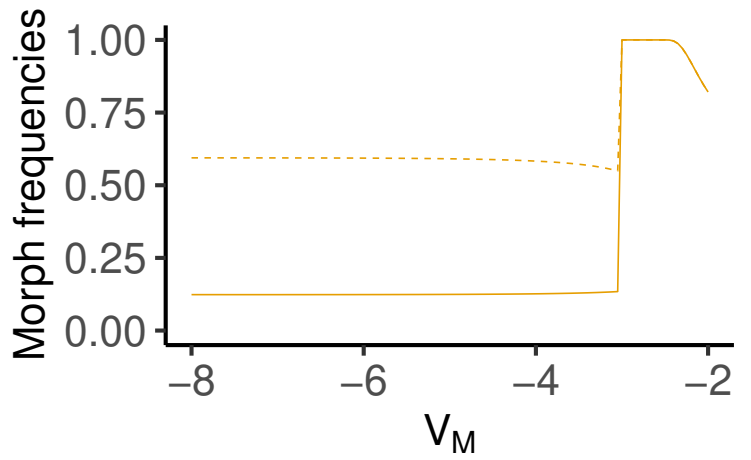

Figure S.6: Effect of mutation. The density distribution in habitats A (panel (a)) and B (panel (b)), and the frequencies of morph 2 in each habitat (panel (c); A: solid, B: dashed) are shown as a function of the mutational variance $V_{M}\left(\log\right.$ scale). When $V_{M}$ increases, the variance of the distributions increases. There is a threshold at $V_{M} \approx 10^{-3}$ above which the oligomorphic approximation breaks down. 
We can then write the vital rates as

$$
\begin{aligned}
& r^{A A}(z)=b-n^{A} \sum_{\ell} f_{\ell}^{A} a\left(z-\bar{z}_{\ell}^{A}\right)-g\left(z-\theta_{A}\right)^{2}-m+O\left(\varepsilon^{2}\right) \\
& r^{B B}(z)=b-n^{B} \sum f_{\ell}^{B} a\left(z-\bar{z}_{\ell}^{B}\right)-g\left(z-\theta_{B}\right)^{2}-m+O\left(\varepsilon^{2}\right)
\end{aligned}
$$

and the partial derivatives:

$$
\begin{aligned}
& \left.\frac{\partial r^{A A}}{\partial z}\right|_{z=\bar{z}_{i}^{A}}=-n^{A} \sum_{\ell} f_{\ell}^{A} a^{\prime}\left(\bar{z}_{i}^{A}-\bar{z}_{\ell}^{A}\right)-2 g\left(\bar{z}_{i}^{A}-\theta_{A}\right)+O\left(\varepsilon^{2}\right) \\
& \left.\frac{\partial^{2} r^{A A}}{\partial z^{2}}\right|_{z=\bar{z}_{i}^{A}}=-n^{B} \sum_{\ell} f_{\ell}^{A} a^{\prime \prime}\left(\bar{z}_{i}^{B}-\bar{z}_{\ell}^{B}\right)-2 g+O\left(\varepsilon^{2}\right)
\end{aligned}
$$

with similar expressions for the partial derivatives in habitat $B$. We can then use these expressions in equations (S.4) to obtain the general oligomorphic approximation of the resource competition model. This is how the numerical simulations in figure 3 in the main text were performed. 